\title{
ANL- -8012067
}

ANL -8012067

DE88 012067

\section{Determination of Temperature and Phase Distrikutions in Irradiated U-Pu-Zr Fuel}

\section{A Thesis}

Presented in Partial Fuifillment of the Requirements for the DEGREE OF MASTER OF SCIENCE

\author{
with a
}

Major in Mechanical Engineering

in the

GRADUATE SCHOOL

UNIVEERSITY OF IDAHO

by

Charles E. Lahm

February 1988

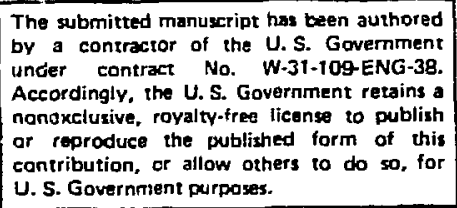

\section{DISCAIMER}

This report was prepared as an account of work sponsored by an agency of the United States Government. Neither the United States Government nor any agency thereof, nor any of their employees, makes any warranty, express or implied, or assumes any iegal liability or responsibility for the accuracy, completeness, or usefulness of any information, apparatus, product, or process disclosed, or represents that its use would not infringe privately owned rights. Reference herein to any specific commercial product, process, or service by trade name, trademark, manufacturer, or otherwise does not necessarily constitute or imply its endorsement, recommendation, or favoring by the United States Government or any agency thereof. The views and opinions of authors expressed herein da not necessarily state or reflect those of the United States Government or any agency thereof.
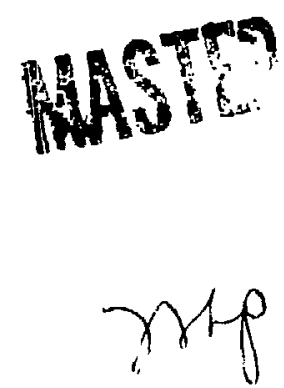


\section{AUTHORIZATION TO SUBMIT \\ THESIS}

This THESIS of Charles E. Lahm, submitted for the degree of MASTER OF SCIENCE with a major in MECHANICAL ENGINEEFING and titled

"Determination of Temperature and Phase Distributions in Irradiated U-Pu-Zr

Fuel," has been reviewed in final form and approved, as indicated by the signatures and dates given below. Permission is now granted to submit final copies to the Graduate School for approval.

Major Professor

Committee Members

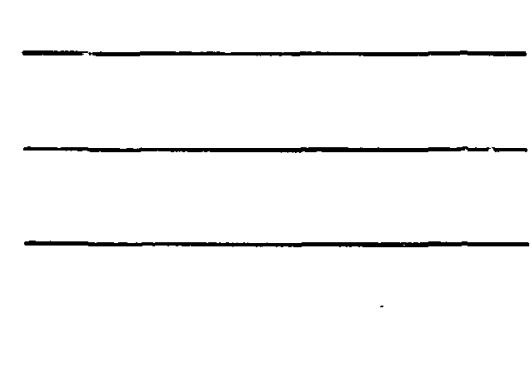

Administrator

College Dean
Date

Date

Date

Date

Date

GRADUATE SCHOOL FINAL APPROY M AND ACCEPTANCE:

Graduate School

Dean

Date 


\begin{abstract}
AESTRACT
Irradiation of LMFBR (Liquid Metal Fast Breeder Reactor) fuel produces significant changes in the thermophysical properties and the structure of the fuel during its lifetime. These changes in properties can be quite large and affect the operating temperatures of the fuel. An experimental subassembly, X423, containing six fuel compositions based on the U J-Pu-Zr system, was irradiated in Experimenial Breeder Reactor -II (EBH-II) and examined at 1 at.\% burnup. Fission gas porosity was measured using an optical image analysis system to provide input for a model of a fuel element analysed with the thermal-hydraulic code THT-B. The model was structured to allow discrete radial fuel compositions and properties based on the measured porosity. This model was subsequently used to determine temperatures and to predict the fuel phases present.
\end{abstract}




\section{ACKNOWLEDGMENTS}

This work was supported by the U. S. Department of Energy, Reactor Systems, Development and Technology, under Contract W-31-109-Eng-38. The author is employed by the EBR-II Division of Argonne National Laboratory in Idaho Falls, Idaho 83403. The author would like to acknowledge the assistance of the personnel of the EBR-II Division and of REE Division at the Hot Fuels Examination Facility who have contributed to these investigations. Several individuals should be recognized for their contributions which have far exceeded their required duties. Dr. D. L. Porter, my advisor and Manager of the Reactor Materials Section who encouraged me to perform the $\mathrm{X} 423$ experiment. Dr. R. G. Pahl, Jr., who provided constructive criticism during all phases of this experiment. J. F. Koenig, who taught me the mechanics of running thermal-hydraulic codes and is a constant source of information about EBR-II. Lasi but not least, R. S. Wisner, who developed the image analysis technique and performed the tedious measurements of each fuel element. 
v

TABLE OF CONTENTS

PAGE

I. INTRODUCTION

II. DESCRIPTION OF EXPERIMENT

III. MEASUREMENT OF POROSITY

IV. METHOD OF TEMPERATURE CALCULATION

V. THERMAL CONDUCTIVITY CALCULATION

VI. RESULTS OF TEMPERATURE CALCULATIONS

VII. FUEL PHASE PREDICTION

VIII. SUMMARY

IX. BIBLIOGRAPHY
1

1

3

4

6

9

10

12

13 


\section{LIST OF FIGURES}

Figure

Page

1. Design of the $X 423$ fuel elemert. 14

2. Element positions in subassembly X423. 15

3. Transverse metallographic section through center of U-26Pu-10Zr element T353.

4. THT-B model of fuel element, including node numbering and dimensions.

5. Axial power distribution in $\mathrm{X} 423$ based on $\mathrm{Nb95}$ gamma scan of U-19Pu-10Zr element T305.

6. Radial porosity measurements, $U-10 Z$ r.

7. Multiplier for thermal conductivity reduction, $U-10 Z r$. 20

8. Radial temperature profiles in fresh fuel model, $\mathrm{U}-10 \mathrm{Zr}$. 21

9. Radial temperature profiles after reducing thermal conductivity in zones $1-9, \mathrm{U}-10 \mathrm{Zr}$.

10. Radial temperature profiles with reduced thermal conductivity in all zones, U-10Zr.

11. Radial porosity measurements, U-3Pu-10Zr.

12. Multiplier for thermal conductivity reduction, U-3Pu-10Zr.

13. Radial temperature profiles in fresh fuel model, U-3Pu-10Zr.

14. Radial temperature profiles after reducing thermal conductivity in zones 1-9, U-3Pu-10Zr.

15. Radial temperature profiles with reduced thermal conductivity in all zones, U-3Pu-10Zr.

16. Radial porosity measurements, U-8Pu-10Zr.

17. Multiplier for thermal conductivity reduction, U-8Pu-10Zi.

18. Radial temperature profiles in fresh fuel model, U-8Pu-10Zr.

19. Radial temperature profiles after reducing thermal conductivity in zones 1-9, U-8Pu-10Zr.

20. Radial temperature profiles with reduced thermal conductivity in all zones, U-8Pu-10Zr.

21. Radial porosity measurements, U-19Pu-10Zr. 34

22. Multiplier for thermal conductivity reduction, U-19Pu-10Zr. 35

23. Radial temperature profiles in fresh fuel model, U-19Pu-10Zr.

24. Radial temperature profiles after reducing thermal conductivity in zones 1-9, U-19Pu-10Zr.

25. Radial temperature profiles with reduced thermal conductivity in all zones, U-19Pu-10Zr.

26. Radial porosity measurements, U-22Pu-10Zr. 


\section{LIST OF FIGURES (Continued)}

Figure

Page

27. Multiplier for thermal conductivity reduction, $\mathrm{U}-22 \mathrm{Pu}-10 \mathrm{Zr}$.

28. Radial temperature profiles in fresh fuel model, U-22Pu-10Zr.

29. Radial temperature profiles after reducing thermal conductivity in zones 1-9, U-22Pu-10Zr.

30. Radial temperature profiles with reduced thermal conductivity in all zones, U-22Pu-10Zr.

31. Radial porosity measurements, U-26Pu-10Zr. 44

32. Multiplier for thermal conductivity reduction, U-26Pu-10Zr. 45

33. Radial temperature profiles in fresh fuel model, U-26Pu-10Zr.

34. Radial temperature profiles after reducing thermal conductivity in zones 1-9, U-26Pu-10Zr.

35. Radial temperature profiles with reduced thermal conductivity in all zones, U-26Pu-10Zr.

36. Possible phases and phase boundaries in $\mathrm{U}-10 \mathrm{Zr}$ element T332.

37. Possible phases and phase boundaries in U-3Pu-10Zr element T347.

38. Possible phases and phase boundaries in U-8Pu-10Zr element T358.

39. Possible phases and phase boundaries in U-19Pu-10Zr element T305.

40. Possible phases and phase boundaries in $\mathrm{U}-22 \mathrm{Pu}-10 \mathrm{Zr}$ element T303.

41. Possible phases and phase boundaries in U-26Pu-10Zr element T353. 


\section{INTRODUCTION}

The fuel currently chosen for use in future Liquid Metal Fast Breeder Reactors (LMFBR) consists of a metallic alloy of uranium, plutonium and zirconium. The amount of plutonium varies with the lifetime of the core as more $\mathrm{U}-238$ is converted to plutonium and becomes available for recycling. Early testing done with the U-Pu-Zr system revealed radial zone formation in the fuel with redistribution of the fuel alloy constituents. In order to better understand the cause of the redistribution, and its possible effect on fuel performance, several tests were initiated.

This experiment was designed to investigate beginning-of-life fuel performance and was conducted in Experimental Breeder Reactor II (EBR-II) in subassembly $X 423$. It was known that metallic fuels swelled when irradiated due to the accumulation of fission products, especially the inert gaseous fission products. This swelling could change the thermal properties of the fuel and contribute to the formation of the radial zones characterized by differing porosity distributions and fuel compositions. It was also assumed that the temperature gracient in the fuel could be a contributor to the redistribution due to the Soret effect ${ }^{1}$, and this was investigated separately. The results of the $X 423$ experiment are presented here for only one burnup level to demonstrate the technique and to provide a plausible explanation of the zone formation. Other results of the experiment have been or will be published elsewhere.

\section{DESCRIPTION OF EXPERIMENT}

The primary objective of this experiment was to irradiate several fuel compositions under similar conditions and perform frequent examinations to characterize and compare the performance. This experiment was irradiated to a total of $=5$ atom percent (at.\%) burnup (burnup is calculated as number of atoms fissioned divided by the number of iritial heavy matal atoms) with reconstitution at $\approx 0.5$ at. $\%, \approx 1.0$ at. $\%$, and $\approx 2.0$ at. $\%$ burnups to allow examination of low-burnup behavior. Six fuel compositions were included: U-10Zr, U-3Pu-10Zr, U-8Pu-10Zr, 
U-19Pu-10Zr, U-22Pu-10Zr and U-26Pu-10Zr, where all compositions are given in weight percent (wt.\%). In order to obtain equal pin powers at beginning of life, the U-235 enrichment was varied for each composition.

The subassembly consisted of 37 cylindrical fuel elements within a 5.817 $\mathrm{cm}$ (2.290 inch), across the flats, hexagonal duct. A $0.142 \mathrm{~cm}$ (0.056 inch) diameter spacer wire is wound helically around each fuel element on a $15.24 \mathrm{~cm}$ (6 inch) pitch to maintain the triangular lattice spacing and to provide mixing of the sodium coolant within the subassembly. The cladding was constructed of 316 stainless steel and was $0.737 \mathrm{~cm}$ (0.290 inches) in outside diameter by $0.655 \mathrm{~cm}$ ( 0.258 inches) inside diameter and $\approx 63.5 \mathrm{~cm}$ (25 inches) long, figure 1 . The element identity was stamped on the endplug and consisted of a three digit number with the prefix $\mathrm{T}$. The element positions in the subassembly are shown in figure 2 with a list of the elements examined and their compositions. The fuel slug was $0.566 \mathrm{~cm}$ (0.223 inches) in diameter and $34.3 \mathrm{~cm}$ (13.5 inches) long and thermally bonded to the cladding with sodium. The fuol slugs were injection cast into precision glass molds which had been coated with a zirconia mold wash to reduce interaction between the mold and the fuel. The plenum region contains an argon-helium mixture at approximately atmospheric pressure and a unique ratio of xenon isotopes which aid in identifying breach of the elements.

The first examination at $=0.5$ at. $\%$ burnup revealed some swelling 2 , but the image analysis technique for determining porosity distribution had not been requested for this burnup. The examination of $X 423 A$ at $\approx 1.0$ at. \% burnup was used to provide data for this thesis. Axial gamma scanning was performed on the six elements selected for destructive examination to determine the axial distribution of various radioactive isotopes. Optical metallography revealed bubble distributions which could be measured to provide input for refinement of the temperature calculations. Sections were also removed for radial chemistry samples to determine the radial distribution of uranium, plutonium and zirconium by wet chemistry analyses. These measurements are described below. 


\section{MEASUREMENT OF POROSITY}

The destructive examination of the fuel elements included sectioning at top, center and bottom for optical metallography. At 1 at.\% burnup the fuel had grown axially from the original $34.3 \mathrm{~cm}$ ( 13.5 inches) to $\approx 35.6 \mathrm{~cm}$ (14.00 inches). The top section was cut at a distance of $33.02 \mathrm{~cm}$ (13.00 inches) above the bottom of the fuel column. The center section was cut at $17.15 \mathrm{~cm}(6.75$ inches) and the bottom section was cut at $1.27 \mathrm{~cm}$ ( 0.50 inches). These sections were then ground and polished for metallography. A montage of $100 \mathrm{x}$ photomicrographs was assembled to show typical fuel structure from the center of the fuel past the cladding.

The optical image analysis was performed using an Omnicon 3500 image analysis system built by Bausch and Lomb. The system includes an automated macroviewer which allows photographs to be incrementally and continuously scanned by a Vidicon scanner. The system is controlled and driven by : Data General Eclipse S-120 minicomputer.

The analysis was done by measuring ten areas approximately $0.033 \mathrm{~cm} \times$ $0.033 \mathrm{~cm}(0.0129$ inches $\times 0.0129$ inches) starting at the measured centerline of the fuel and going outward to the fuel cladding inner diameter. The areas continuously covered the distance from centerline to fuel cladding inner diameter without gaps or overlapping. The pores in each frame were counted 10 times to allow slight adjustments of grey level thresholding to detect the entire range of pores, some of which appeared lighter or darker than others. The pore size data was collected and fit into two histograms. One histogram was linear in distribution with 20 classes starting from 0.0 to $3.2 \times 10^{-8} \mathrm{~cm}^{2}\left(0.0\right.$ to $0.5 \times 10^{-8}$ square inches) and incrementing each subsequent class by $3.2 \times 10^{-8} \mathrm{~cm}^{2}(0.5$ $\times 10^{-8}$ square inches) with the last class of pore sizes being from 61.3 to $64.5 \times$ $10^{-8} \mathrm{~cm}^{2}$ (9.5 to $10.0 \times 10^{-8}$ square inches). The second histogram had 20 classes starting from 1.9 to $29.0 \times 10^{-8} \mathrm{~cm}^{2}$ (0.3 to $4.5 \times 10^{-8}$ square inches) incrementing each successive class by a factor of 1.5 , with the last class being pore sizes between 4290.3 to $6436.1 \times 10^{-8} \mathrm{~cm}^{2}$ (665.0 to $997.6 \times 10^{-8}$ square 
inches). These ranges were chosen to allow classification of the smaller pores while allowing sufficient range to include most if not all of the largest pores. Additionally, statistics were provided for all pores in order to include even the largest ones not otherwise classified by the logarithmic distribution. After all 10 areas were scanned a summary was printed which included the number of features, total area, minimum feature size and maximum feature size. Both the number of features and total area for each scan were divided by 10 to obtain the actual measurement for any given zone.

There were two problems which occurred when using the scanner to measure porosity. The first occurred because the fuel did not entirely fill the cladding and left a gap between the fuel and cladding which was measured as a very large void. This gap could be filled with sodium or gas or both when in-reactor, and was summed as part of the area measured for that zone. This zone could be modeled in greater detail based on the appearance in the micrograph, but the uncertainties in the properties in this zone would not be reduced. Adjacent sectors may be different in both size and material visible in the zone and detailed modeling of the entire circumference is not possible. The second problem occurred when the pore count was very high. This required that the frame size be reduced by only using half the width.

A typical example of the metallography used for the porosity measurements is shown in figure 3. This is the certer section of the U-26Pu-10 Zr element T353. The gap between the fuel and cladding and the various zones of porosity can be seen.

\section{METHOD OF TEMPERATURE CALCULATION}

The thermal-hydraulic code used to calculate the temperatures in this experiment was THT-B. This is a general three-dimensional transient heat transfer code using a finite difference method which was developed by General Electric and modified for use at Argonne National Laboratory. The program sets up the general heat balance equation for each node point and solves the 
resulting set of equations for the central temperature of the node using the Gauss-Seidel method. Material properties are lineariy interpolated from tables adied as input to the code. The cylindrical geometry option was used for this analysis. The code allows an option of using either the average radius or the centroid of the node for the point of temperature calculation. In this analysis the temperature is calculated for the centroid of the node. The node width was chosen arbitrarily small ( 1 degree) to minimize the difference beiween centroid location and geometric center (average radius) of the node. For critica! temperatures such as fuel certerline or cladding inside surface, small nodes were used to provide a centroid closer to the location of interest. The geometry of the model is shown in figure 4.

The probiem cf caiculating temperatures in fuel elements is quite straight-forward at beginning of life, but becomes much more complicated afier very short periods of irradiation. This is because fuel swelling changes both the geomistry and the material properties. At higher bumups the addition of fission products to the fuel may contribute signiricantly to the changes in material properties. Generally, the beginning of life fuel properties and geometry are used during the initial design phase of an experiment, and those calculations are sufficient for safety analyses and estimation of operating conditions. This is especially true for metal fuels where the temperature of the cladding is generally more important in the safety analysis than the temperatures in the fuel.

Very littie data are available for the fuel alloys used in this experiment. In the case of U-PU-Zr, and U-Zr where properties have been measured on only a few alloys, the data have been extrapolated for the alloys being tested. This extrapolation has a recommended uncertainty of $20 \%$ for unirradiated fuel.

The axial power generation portion of the model was determined by using the gamma scan data from the U-19PU-10Zr element T305. Gamma scanning consists oi counting gamma rays of various energies emitted by the fuel element, and correlating the number of gamma rays of each energy to the amount of an isotope which decays with the release of gamma rays of similar energy. Of the isotopes measured, Nb95 is a fission product which is not present in unirradiated 
fuel and not soluble in sodium and provides a goud indication of the relative axial fission rates and the resultant fuel element power. The Nb95 data, taken every $0.05 \mathrm{~cm}(0.02$ inches), was used to obtain a fifth order polynomial fit for the relative axial power distribution. The subassembly power from the EBR-II post-run report was used to obtain the average element power. This was then used in the mcdel based on the curve fit to calculate the power generation rate for each node. All radial nodes at each elevation have the same generation rate and no aitempt was made to adjust radial generation rates based on porcsity measurements. The axial power profile is shown in figure 5.

\section{THERMAL CONDUCTIVITY CALCULATION}

Thie properties of irradiated fuel are difficult to measure and involve using hot cell facilities to handle the highly radioactive fuel. Thermal conductivity cannot be directly measured in the hot cells, but cptical metallography can be used to measure porosity distribution, and this can be used to estimate the thermal conductivity of the fuel. This mettiod is based on theoretical models of porous media and on some measurements made on irradiated fuel. Porosity fraction $(P)$ is defined as the void area divided by the total area. This has resulted in the following equation for determining effective thermal conductivity based on measured porosity 3 :

$$
f_{p}=k / k_{0}=(1-P) /(1+\beta P)
$$

where $P$ is porosity fraction and $1.5 \leq \beta \leq 2.5$

The minimum value of $f_{p}$, based on the uniform expansion of the fuel to fill the cladding, is 0.5 . Because the porosity was measured for this experiment, this was used to reduce the conductivity to values below this minimum value. Studies of porous media don't provide for a minimum value until the thermal conductivity of the gas is reached 4 . This is much lower than the value for the fuel. For example, the thermal conductivity of helium is $\approx 1 / 100$ of that of unirradiated fuel, and the conductivity of xenon and krypton is $<1 / 15$ that of helium.

The initial thermal conductivities for the various compositions were derived 
from an empirical equation for the U-Pu-Zr system which had a recommended uncertainty of $20 \%$. The thermal conductivity decreases with the addition of plutonium. Property tables were generated to simulate various pcrosities by decreasing thermal conductivity in $10 \%$ increments to a low of $20 \%$ of the original value. The model for each element was then changed to use the properties which were closest to the decrease in thermal conductivity predicted by the measured porosity. This provided appropriate properties for the first nine radial zones of fuel.

The greatest uncertainty in the modeling was at the interface between the fuel and cladding. Because the gap was quite large in some elements, there was the possibility that it was completely filled with sodium and would then have a much higher thermal conductivity than the fuel (the thermal conductivity of sodium is $\approx 4$ times that of unirradiated fuel). There was also the possibility that gas which had been released to the surface of the fuel slug would remain there and form bubbles which may or may not rise to the plenum area. Studies with instrumented subassemblies have indicated that fuel temperatures fluctuate and it has been assumed that this is due to bubble formation and migration 5 . Another uncertainty occurs when a layer of mold wash $\left(\mathrm{ZrO}_{2}\right)$ is visible on the surface of the fuel. The thermal conductivity of $\mathrm{ZrO}_{2}$ is $\approx 1 / 10$ that of unirradiated fuel, and thick layers can be seen in some micrographs. This can be modeled by refining the node mesh on the surface of the fuel and including appropriate material properties, and has been done for other subassemblies. For smaller diameter elements, the fuel centerline temperatures were increased by $\approx 35^{\circ} \mathrm{C} / 0.025 \mathrm{~mm}$ $\left(63^{\circ} \mathrm{F} / 0.001\right.$ inch) of $\mathrm{ZrO}_{2}$ added to the surface. For irregular or broken layers of mold wash the uncertainty in modeling is quite large, so this feature was not added to the model. Because of the uncertainties involved in modeling the zone which includes the fuel/cladding interface, three models were analysed with the goal of defining the minimum and maximum operating temperatures for the fuel and one intermediate estimate based on an assumption of properties in that zone. 
The first model used the properties of unirradiated fuel in all ten zones and provides the lower bound of fuel temperatures. Another model was constructed which used the measured porosity to reduce the thermal conductivity of the first nine radial zones, but allowed the thermal conductivity of the last zone to be that of unirradiated fuel. This thermal conductivity is not as high as the value for sodium ( $\approx 4$ times higher), but provides for the possible case of some gas and some scdium. This model is reasonable for this burnup and represents a Dossible temperature profile for the elements. The properties which are used for the zone containing the fuel/clad interface cannot be determined exacily and rnay vary greatly with both location and time. For this study, a maximum fuel temperature was desired, so one model used the thermal conductivity which would be predicted based on the Omnicon measurement of the last zone of fuel. The maximum reduction of thermal conductivity was taken as $80 \%$, even when an actual measurement of the lack of fuel in that zone indicated a higher reduction. This corresponds to the maximum reduction in thermal conductivity used in the interior zones. This model accounts for the possibility of gas or mold wash in the gap acting to insulate the fuel surface.

The axial variation in properties, over the $35.6 \mathrm{~cm}$ (14.0 inch) fuel column, was modeled as follows. The metallography taken at an elevation of $\approx 1.3 \mathrm{~cm}(0.5$ inches) provided the properties for the first $5 \mathrm{~cm}$ (2 inches) of fuel $(0.0$ to $5.0 \mathrm{~cm}$ or 0.0 to 2.0 inches). The metallography taken at an elevation of $\approx 33 \mathrm{~cm}$ (13 inches) provided the properties for the last $5 \mathrm{~cm}$ (2 inches) of fuel ( 30.5 to $35.6 \mathrm{~cm}$ or 12.0 to 14.0 inches). The metallography taken at an elevation of $=17.15 \mathrm{~cm}(6.75$ inches) provided the properties for all the remaining fuel in the center of the element (5.0 to $30.5 \mathrm{~cm}$ or 2.0-12.0 inches). This caused discontinuities in the calculated axial temperature profile at the point where the properties changed, but there were only three samples available to estimate the properties. A more refined model would require additional sections of the fuel element to be cut for metallography.

The results of the analyses are shown in figures 6-35. For each element examined there are plots of the measured porosity, thermal conductivity 
correction factor $f_{p}$, fuel temperatures based on beginning of life thermal conductivity, fuel temperatures based on measured porosity with zone ten modeled as tresh fuel and fuel temperatures based on measured porosity with zone ten modeled as measured or at a minimum of $20 \%$ of uriginal thermal conductivity.

\section{RESULTS OF TEMPERATURE CALCULATIONS}

The results of the temperature calculations were surprising in some respects. The calculated temperature profile based on fresh fuel thermal conductivity was typical of homogeneous materials with heat generation. The coolant and cladding temperatures are similar for each element and for each model because identical power and flow were used in the analyses. Because the power generation decreases at the top of the fuel column, the fuel centerline temperatures also drop off at the top of the element when the thermal conductivity of the fuel is lowared. For the unirradiated fuel model, this occurs when Pu concentration is greater than $3 \mathrm{wt} . \%$. When the conductivity is decreased due to irradiation this decrease in fuel centerline temperature is alsc seen but it depends on the relative porosity of the axial and radial zones. A surprising prediction, which only occurred for two alloys ( $19 \% \mathrm{Pu}$ and $26 \% \mathrm{Pu}$ ), was that the temperatures at the bottom of the fuel element were higher than the temperatures at the top. This occurs when there is a combination of low conductivity at the bottom and center and high conductivity at the top in the last zone of fuel adjacent to the clad. This could possibly occur if either mold wash or gas blanketed a portion of the fuel surface. The last zone of fuel (zone 10) also may be hotter in the center of the column than the top even though the cladding shows a continuously increasing temperature. This is due to the temperature being calculated for the centroid of the zone rather than the fuel surface.

The results of the temperature calculations indicate that the operating temperatures of the fuel can vary greatly after the fuel begins to swell. This is especially true when plutonium is added because it decreases the thermal 
conductivity at beginning-of-life. The temperatures calculated based on the measured porosity are useful for explaining the zone formation in the ternary alloy. Both higher temperatures and higher temperature gradients exist to enhance transport of constituents. It is also possible to have more phases present in the fuel when the higher temperatures exist, which allows different compositions to exist.

\section{FUEL PHASE PREDICTION}

The irradiation of U-Pu-Zr fuels produces a fuel restructured by means of constituent migration, notably zirconium, which contains distinct annular zones. In order to understand why these zones form, several experiments have been conducted and theoretical studies carried out. Thermal transport of zirconium appears to be a very small part of the restructuring if it contributes at all. The major driving force may be due to the creation of radial regions representing specific U-Pu-Zr phase fields in the fuel. Diffusion is driven by the presence of phases with wide variations in $\mathrm{Zr}$ solubility. To identify these phase fields, the temperatures have to be known. There are still uncertainties in the calculated temperatures, but it appears we are able to bound the temperature regimes, and this has been sufficient for identifying the phases which may exist. The phases in the U-Pu-Zr system can be obtained from ternary phase diagrams for various temperatures.

The following phases have been identified in the U-Pu-Zr system. 6 The $\gamma$ phase is a body-centered cubic phase showing complete miscibility for $U(\gamma)$, $\operatorname{Pu}(\varepsilon)$, and $\operatorname{Zr}(\beta)$. The designations $\gamma 1$ and $\gamma 2$ indicate the uranium-rich and zirconium-rich modifications of the $\gamma$ phase. The $\alpha$ and $\beta$ phases are orthorhombic and tetragonal uranium allotropes dissolving up to 15 and 2.0 at.\% Pu respectively, but little $\mathrm{Zr}$. The $\eta$ pinase is a tetragonal intermediate phase in the U-Pu binary with limited solubility for $Z$ r. The $\zeta$ phase is a tetragonal 
intermediate phase in the U-Pu system with up to 5 at.\% solubility for $\mathrm{Zr}$. The $\delta$ phase is a hexagonal phase in the U-Zr system with extensive solid solubility for $\mathrm{Pu}$, but because it is an ordered alloy the $\mathrm{Zr}$ activity is low, despite the high concentration allowed. $\mathrm{APu}(\delta)$ phase is possible as a face-centered cubic $\mathrm{Pu}$ phase with extensive solid solubility for $\mathrm{Zr}$ but limited solubility for $\mathrm{U}$. The cuntribution of each of these phases is dependent on temperature and relative concentration of the constituents.

The low iemperature phases are $\delta$ and $\alpha$ for the low plutonium alloys with the $\zeta$ phase appearing in alloy's with $19 w t . \%$ or more Pu. Of these phases only the $\delta$ phase has significant solubility for $Z r$. At higher temperatures, the $\gamma$ phase has high solubility for zirconium. Of the phases which occur, the transition to the $\gamma$ phase appears to be the most impurtant when investigating the redistribution of zirconium. This is basad on the high zirconium solubility in the $\gamma$ phase. The transition from the $\delta$ phase to the $\gamma$ phase, both of which have high solubility for zirconium, includes various phases with low solubiliiy for zirconium. These low solubility phases may contribute to the redistribution of the zirconium towards the center and outer surface of the fuel slug. The phases identified in these fuel elements at top center and bottom are shown in figures 36-41.

The three columns can be viewed as representing fuel conditions as they vary from beginning of life to current conditions. The beginning of life fuel conductivities are high, and the phases present are the low temperature phases as shown in the first column. With the continuing swelling of the fuel, the thermal conductivity decreases and the transition to the high temperature phases may begin as seen in the second column. If the interface between the fuel and cladding contains gas or mo!d wash, the transition to the phases shown in the third column can occur.

At phase boundaries, changes in fuel properties may become apparent in the metallography. For example, comparing figure 3 to the phase boundaries in 
the center section in figure 41 , the fuel structure appears to change at the predicted phase boundary. The various structural changes or changes in contrast in the metallography however, are not necessarily changes in compositions, although this may occur after continuing irradiation. The radial chemistry analyses performed on this element indicated uniform but elevated $\left(\approx 1.5 \%\right.$ increase) plutonium concentrations from the center to an $R / R_{0}$ of 0.5 . One more sample was taken at $R / R_{0}$ of 0.7 and showed a decrease of plutonium of $\approx 2$ weight percent below the nominal composition. The zirconium distribution was not as uniform as the plutonium, but indicated a decrease from the center to an $R / R_{0}$ of 0.4 of $\approx 1 \%$ below the nominal, no change at $R / R_{0}$ of 0.5 , and an increase of $=1 \%$ above the nominal composition at $R / R_{0}$ of 0.7 . These changes are very small when compared to other tests examined at higher burnups and may indicate that the porosity is formed, decreasing the thermal conductivity, before the redistribution of the constituents occurs.

\section{SUMMARY}

The irradiation of U-Pu-Zr fuels rapidly produces changes in fuel properties and eventually the formation of distinct annular zones of varying composition. The chanye in thermal conductivity due to the formation of fission gas bubbles in the fuel produces much higher fuel temperatures and allows the existence of higher temperature phases. The presence of fission gas or mold wash at the fuel cladding interface may act to increase fuel temperatures even further. These elevated temperatures apparently exist very ea ity in life and exist prior to any major redistribution of the fuel alloy constituents. The higher temperatures may allow the presence of high temperature phases over much of the fuel column and could explain the cause of zirconium migration. The measurement of porosity using the optical image analysis system appears to be a valuable method for adjusting fuel properties for post irradiation analyses. 


\section{BIBLIOGRAPHY}

1. R. B. Bird, W. E. Stewart, E. N. Lightfoot, Transport Phenomena, John Wiley \& Sons, 1960. p 567.

2. C. E. Lahm and R. G. Pahl, Swelling of Metallic Fuels, Am. Cer. Soc. 1987 Annual Meeting, April 28, 1987, Pittsburgh, Pa.

3. A. Biancheria, The Effect of Porosity on Thermal Conductivity of Ceramic Bodies, Trans. Am. Nucl. Soc. 9(1), 15 (1966).

4. W. M. Rohsenow, Handbook of Heat Transfer, McGraw-Hill, 1973.

5. P. R. Betten, In-core Measurements of U-5 wt \% Fissium Alloy Thermal Conductivity, Am. Nucl. Soc. 1985 Winter Annual Meeting, Nov.10-14, 1985, San Francisco, California.

6. D.R. O'Boyle and A. E. Dwight, The Uranium-Plutonium-Zirconium Ternary Alloy System, Plutonium 1970 And Other Actinides, W. N. Miner editor, Nuclear Metallurgy, Volume 17, 1970. 


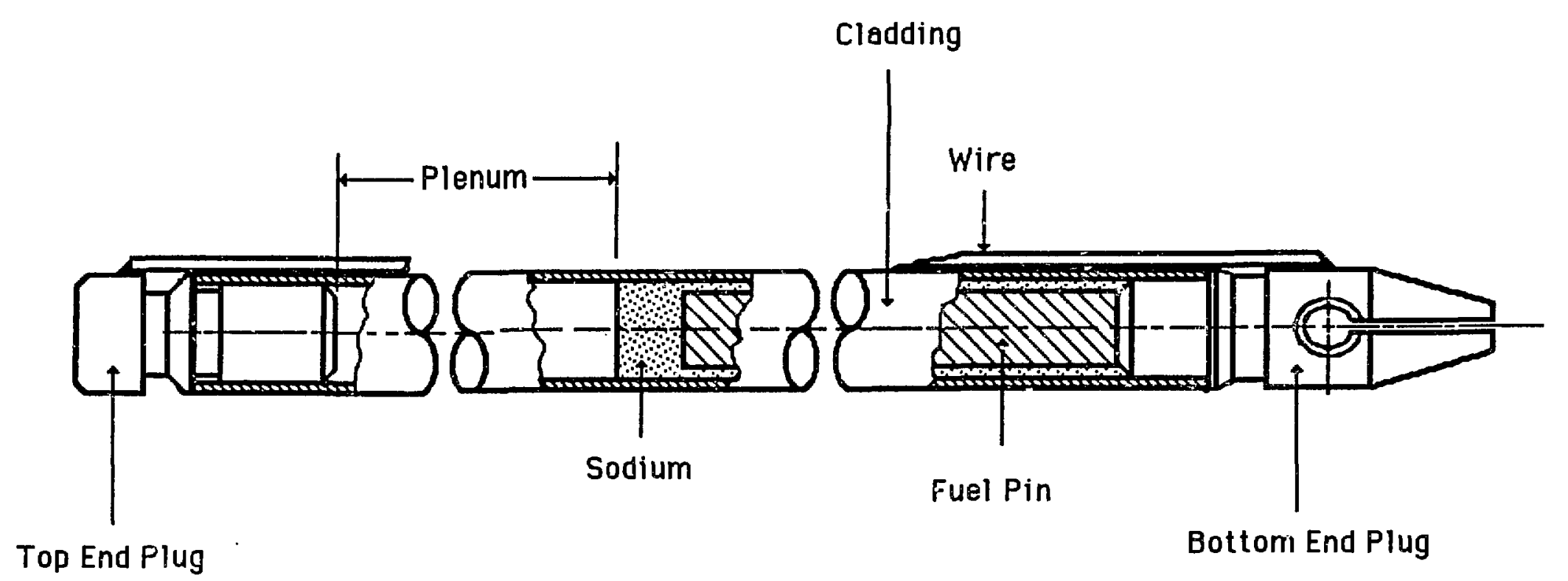

Figure 1. Design of the $X 423$ fuel element. 


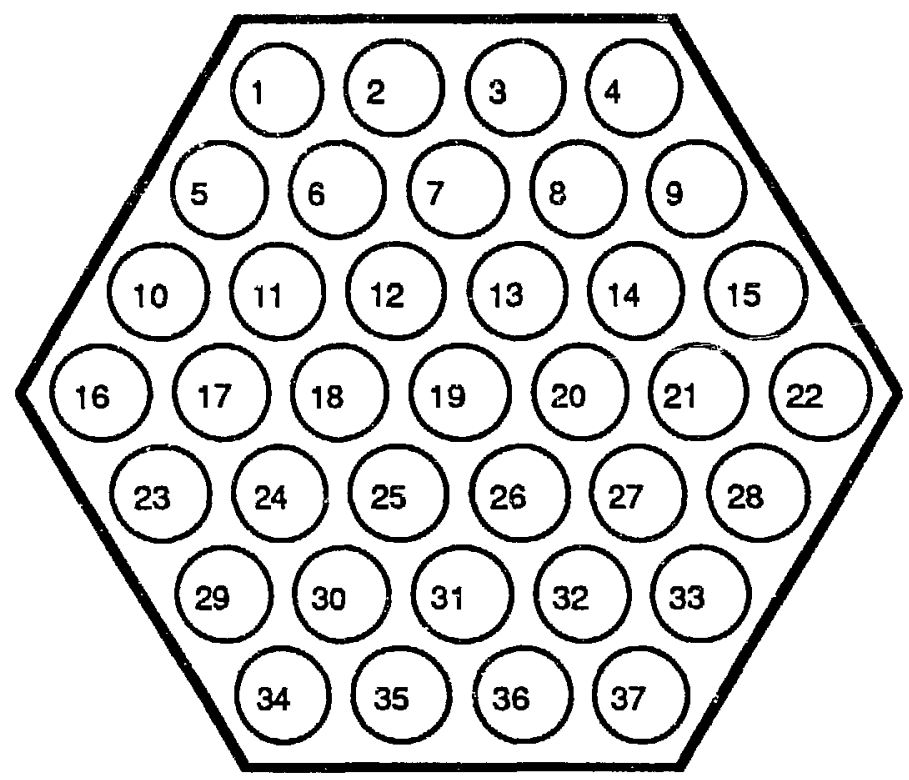

Element Position

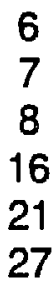

Identity

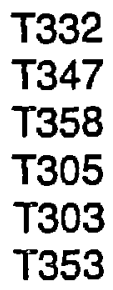

Composition

$U-10 Z r$

$\mathrm{U}-3 \mathrm{Pu}-10 \mathrm{Zr}$

$\mathrm{U}-8 \mathrm{Pu}-10 \mathrm{Zr}$

U-19Pu-10Zr

$\mathrm{U}-22 \mathrm{Pu}-10 \mathrm{Zr}$

$\mathrm{U}-26 \mathrm{Pu}-10 \mathrm{Zr}$

Figure 2. Element positions in subassembly $\mathrm{X} 423$. 


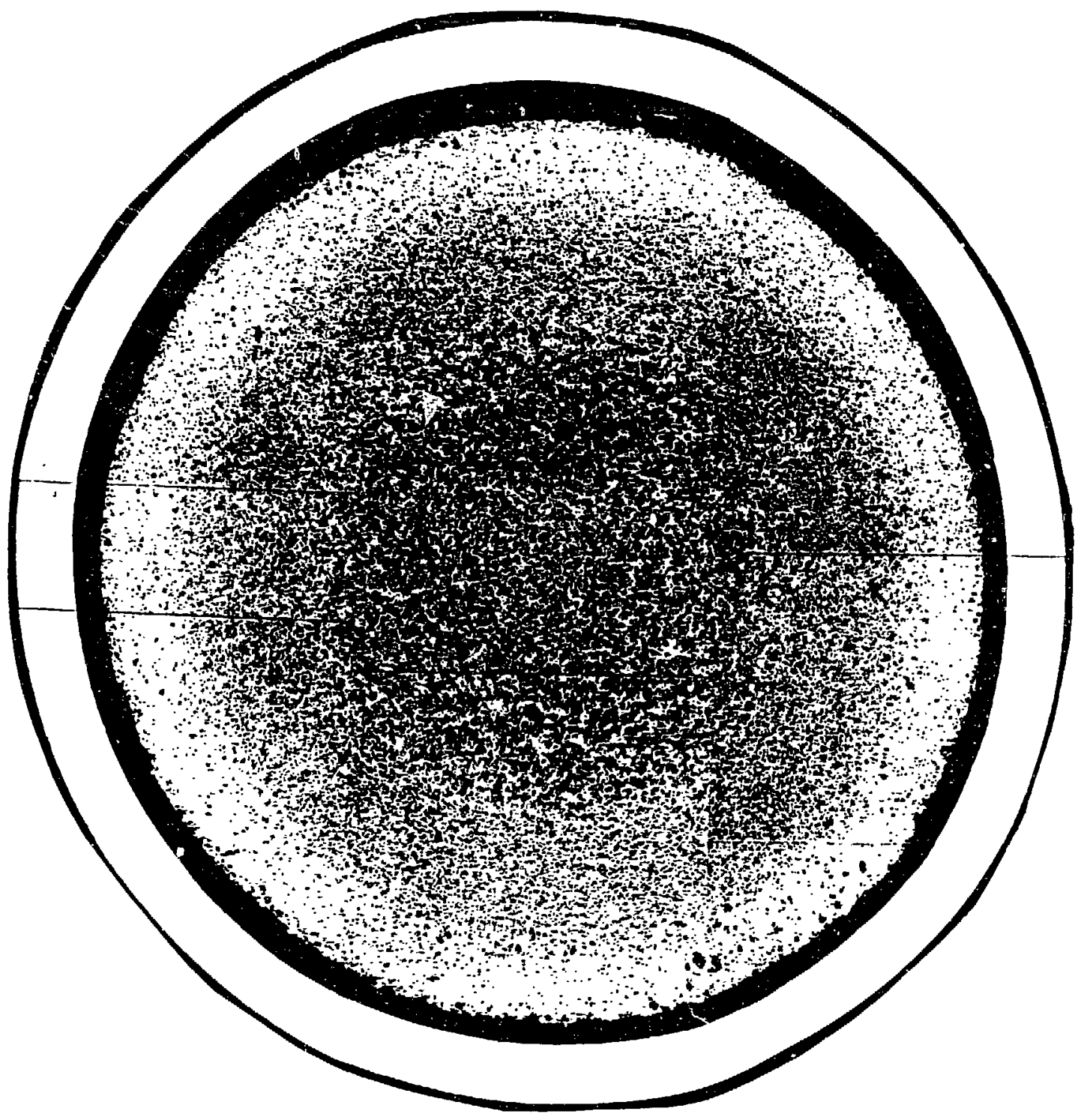

Figure 3. Transverse metallographic section thiough center of U-26Pu-10Zr element T353. 


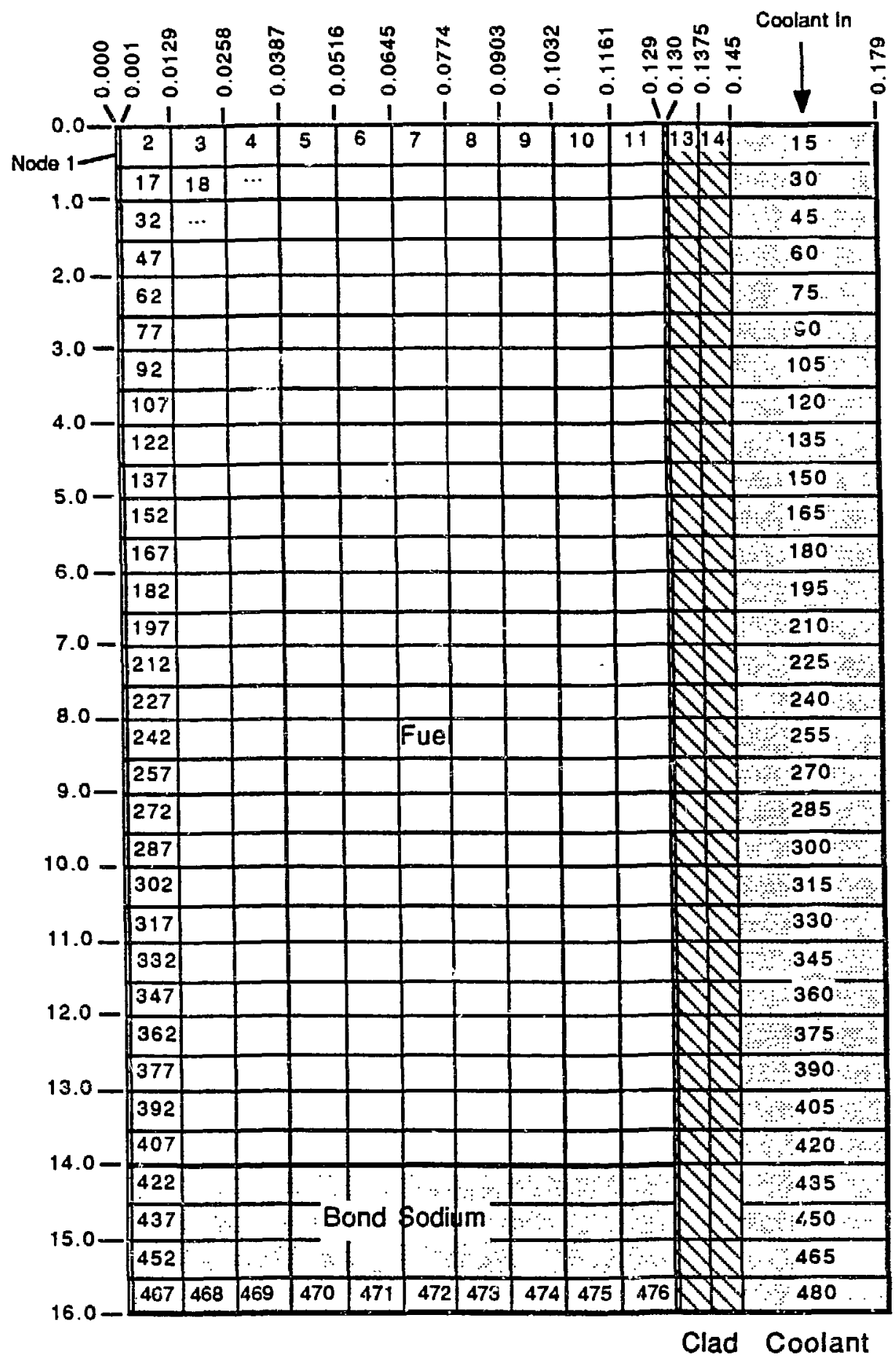

Figure 4. THT-B model of fuel element, including node numbering and dimensions. 


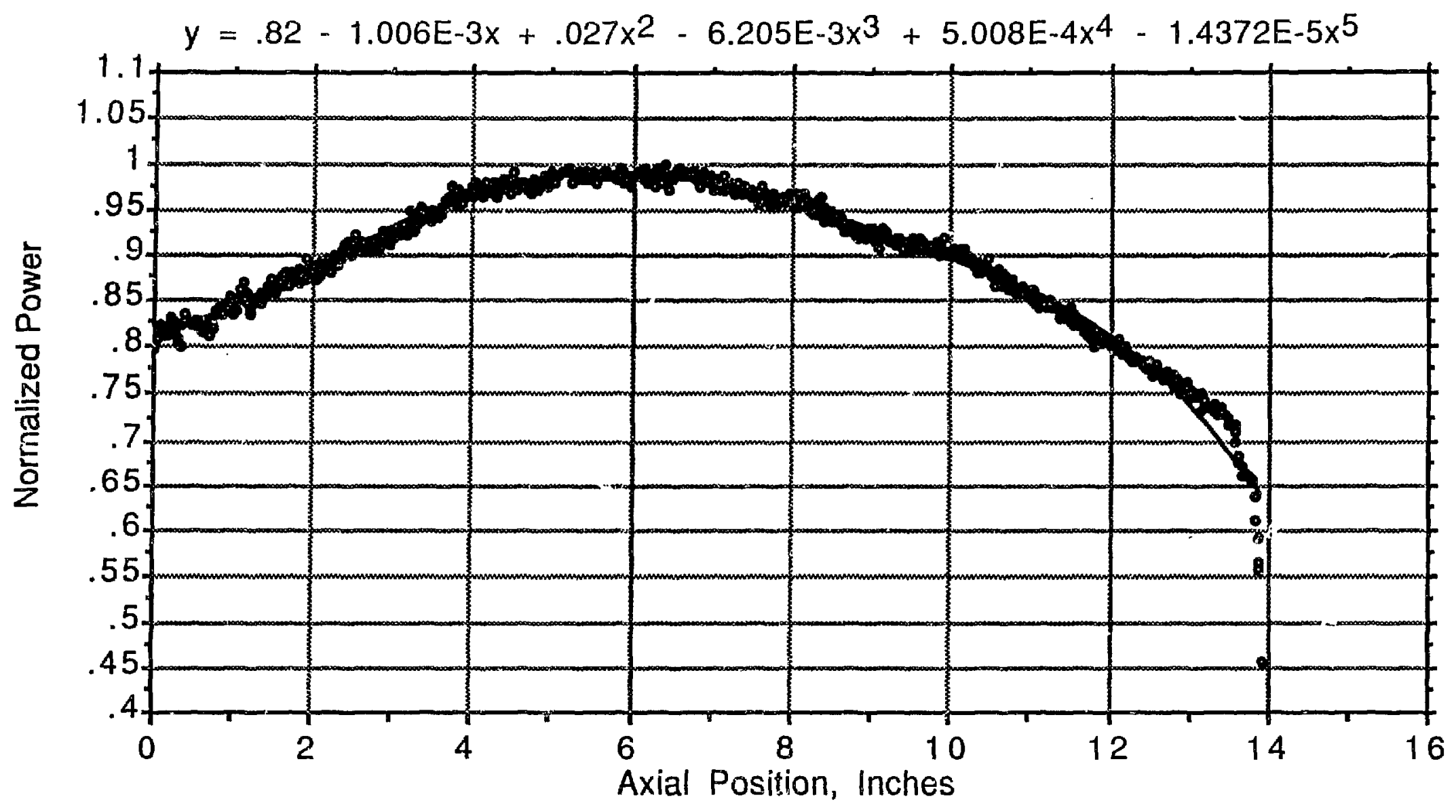

Figure 5. Axial power distribution in $X 423$ based on Nb95 gamma scan of U-19PU-10Zr element T305. 


\section{X423A T332 \\ $U-10 Z r$}

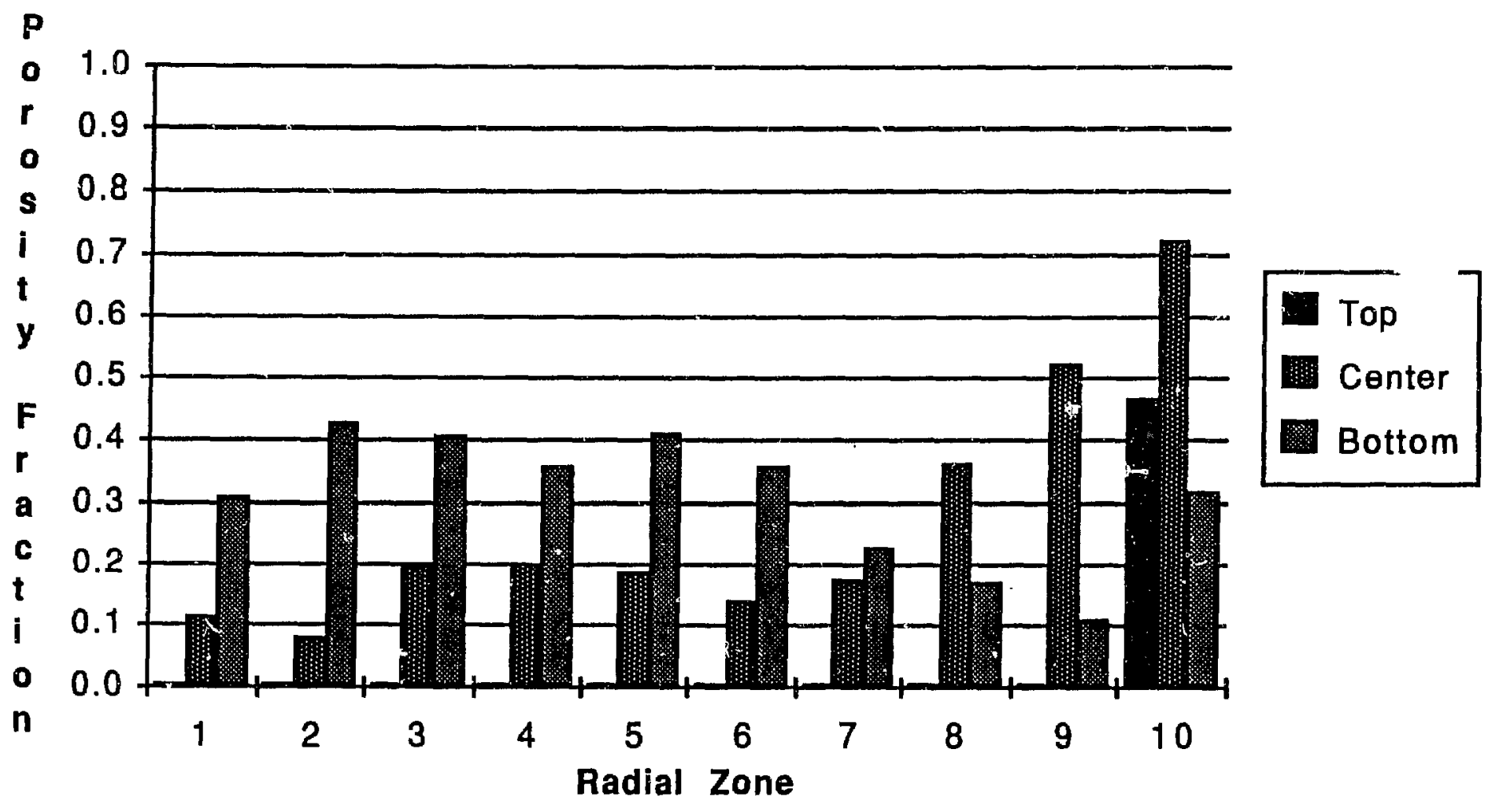

Figure 6. Radial porosity measurements, U-10Zr. 


\section{X423A T332 $\mathrm{U}-10 \mathrm{Zr}$}

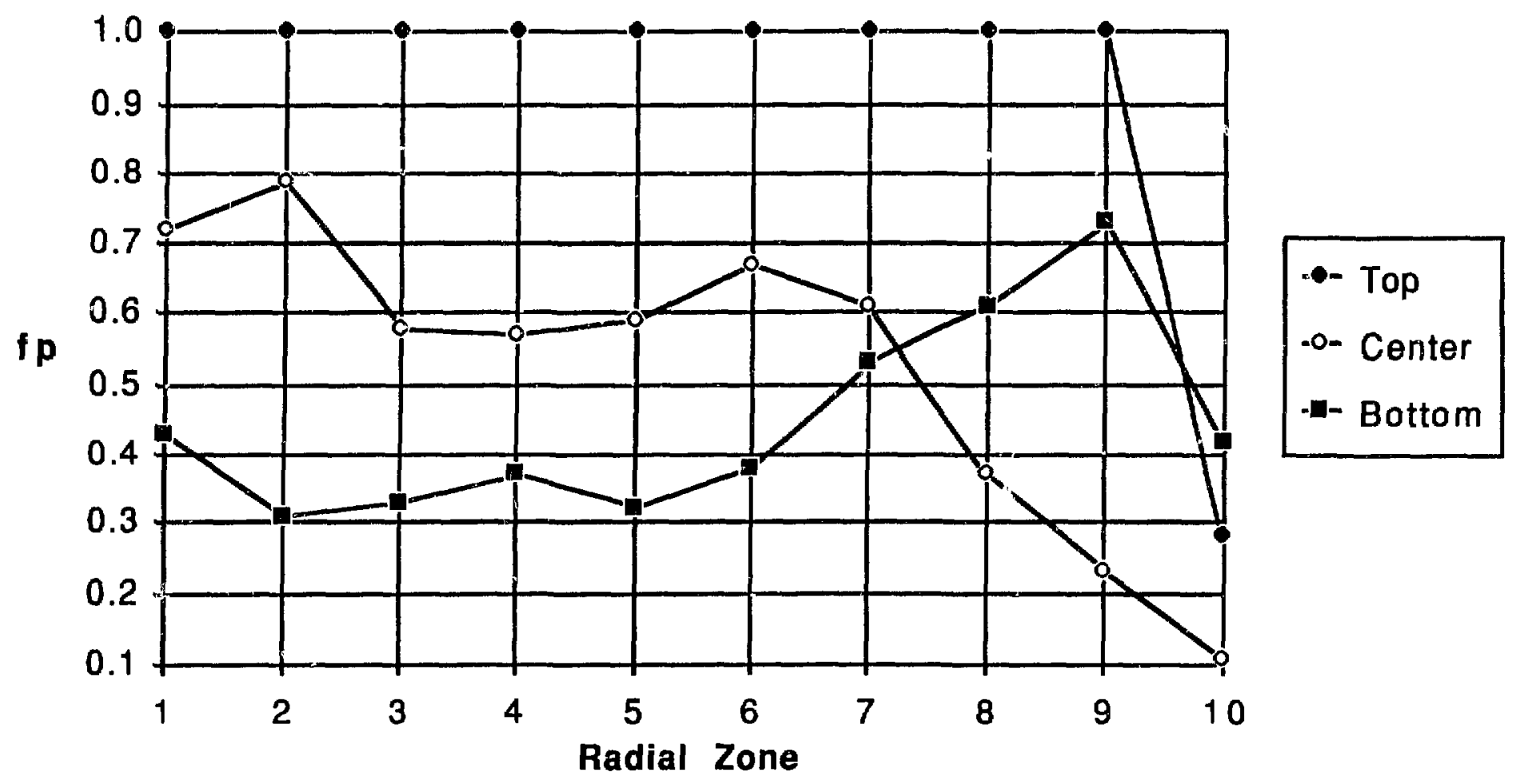

Figure 7. Multiplier for thermal conductivity reduction, U-10Zr. 


\section{X423A T332 $\mathrm{U}-10 \mathrm{Zr}$}

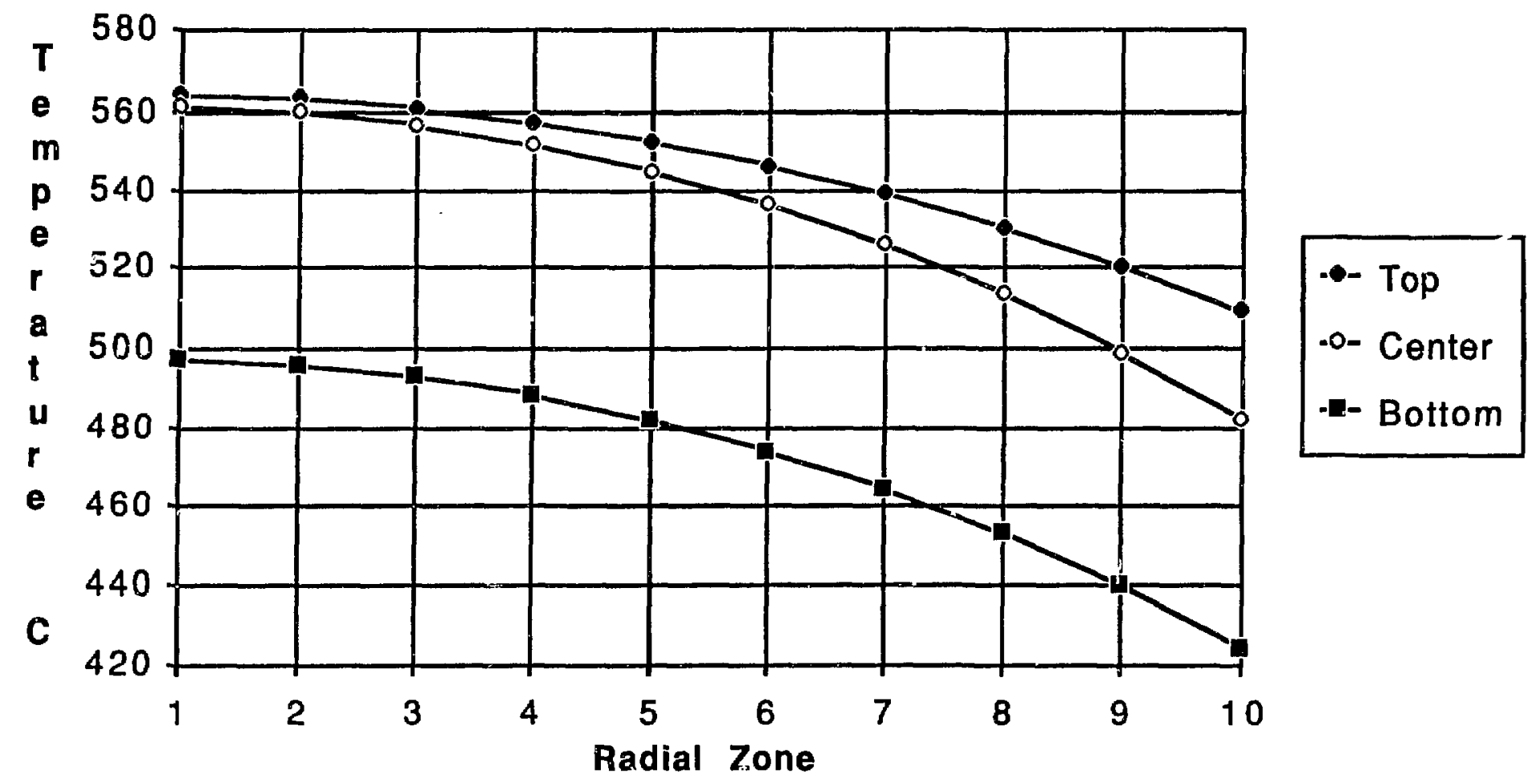

Figure 8. Radial temperature profiles in fresh fuel model, U-10Zr. 


\section{X423A T332 \\ $\mathrm{U}-10 \mathrm{Zr}$}

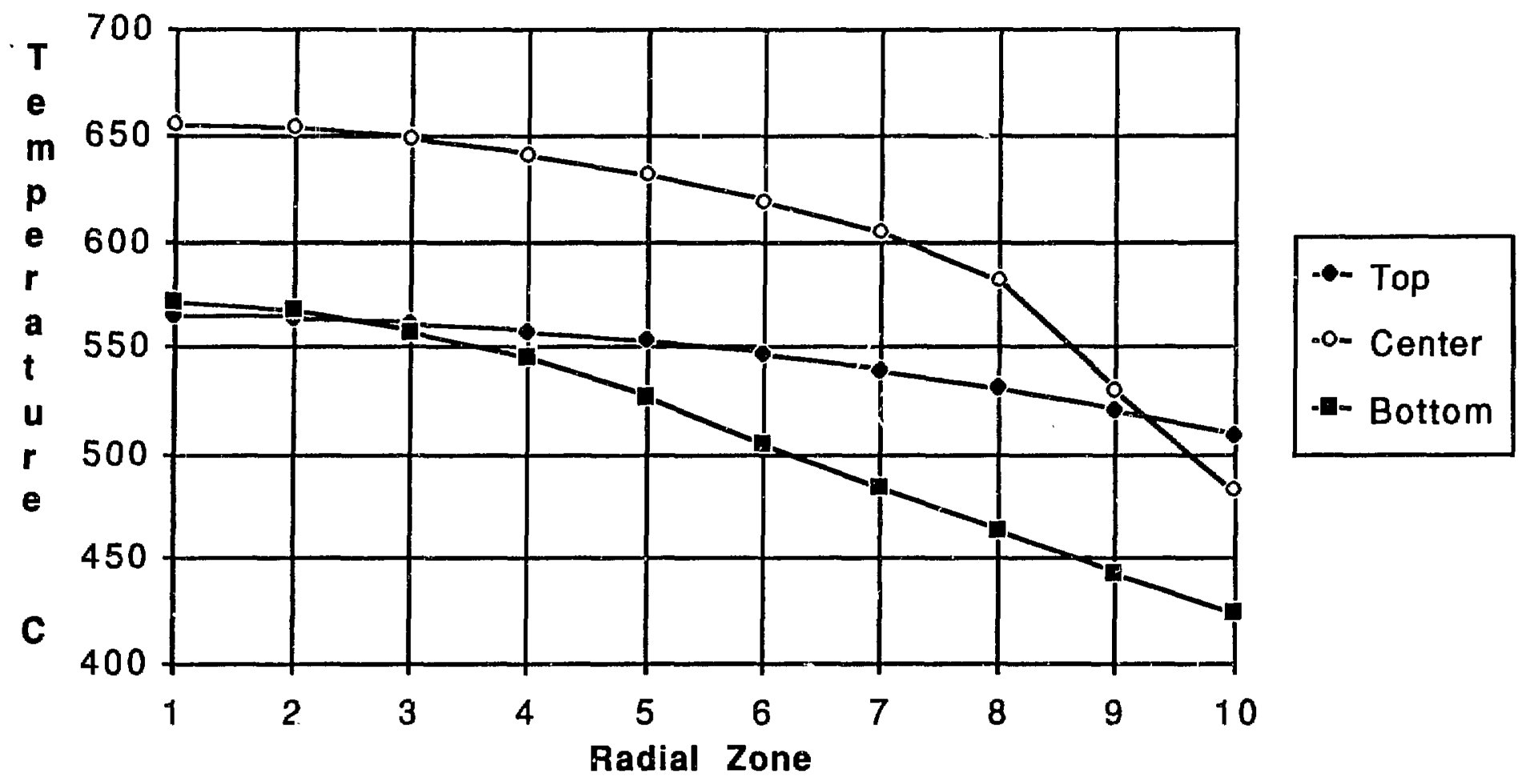

Figure 9. Radial temperature profiles after reducing thermal conductivity in zones 1-9, U-10Zr. 


\section{X423A T332 \\ $\mathrm{U}-10 \mathrm{Zr}$}

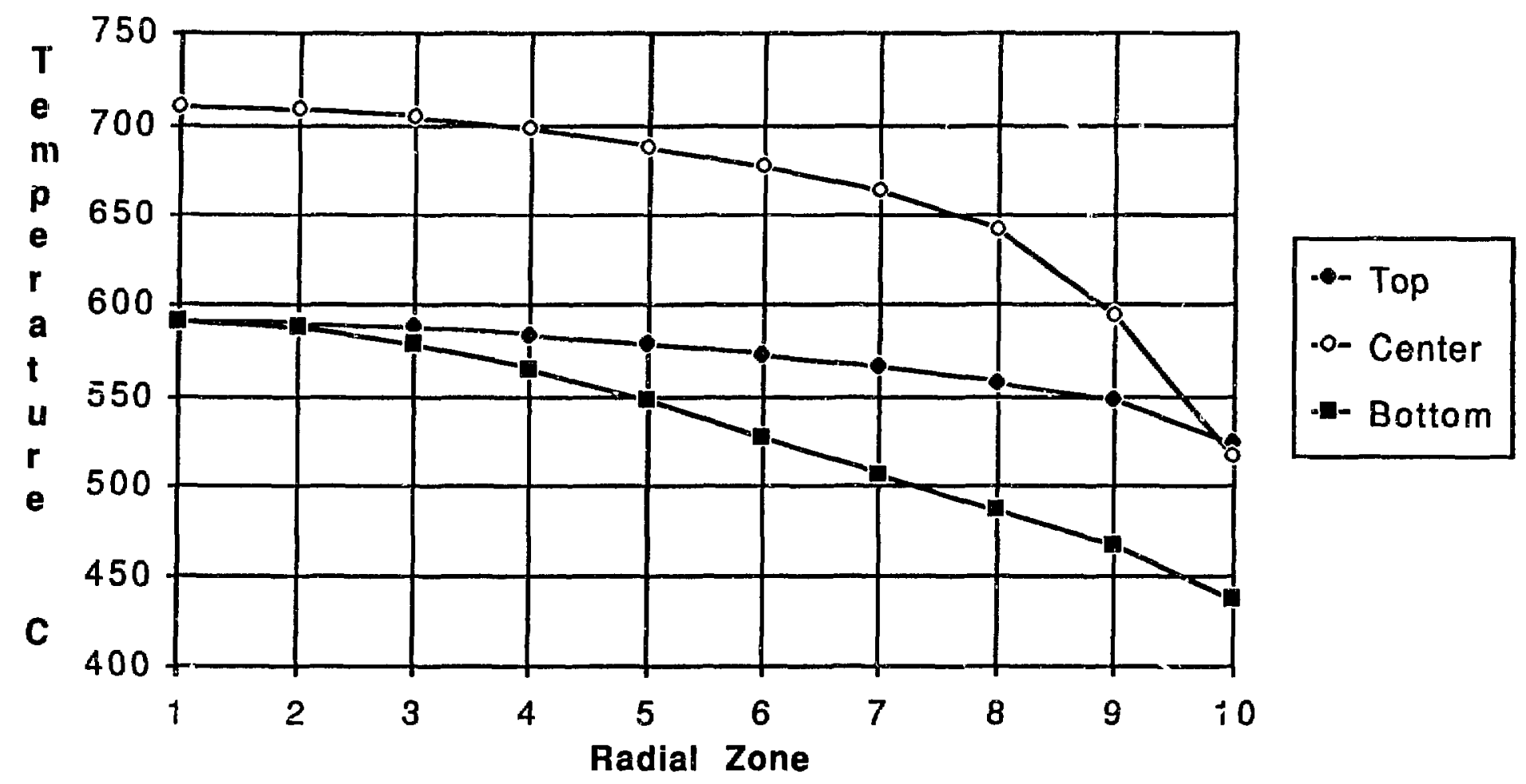

Figure 10. Radial temperature profiles with reduced thermal conductivity in all zones, U-10Zr. 


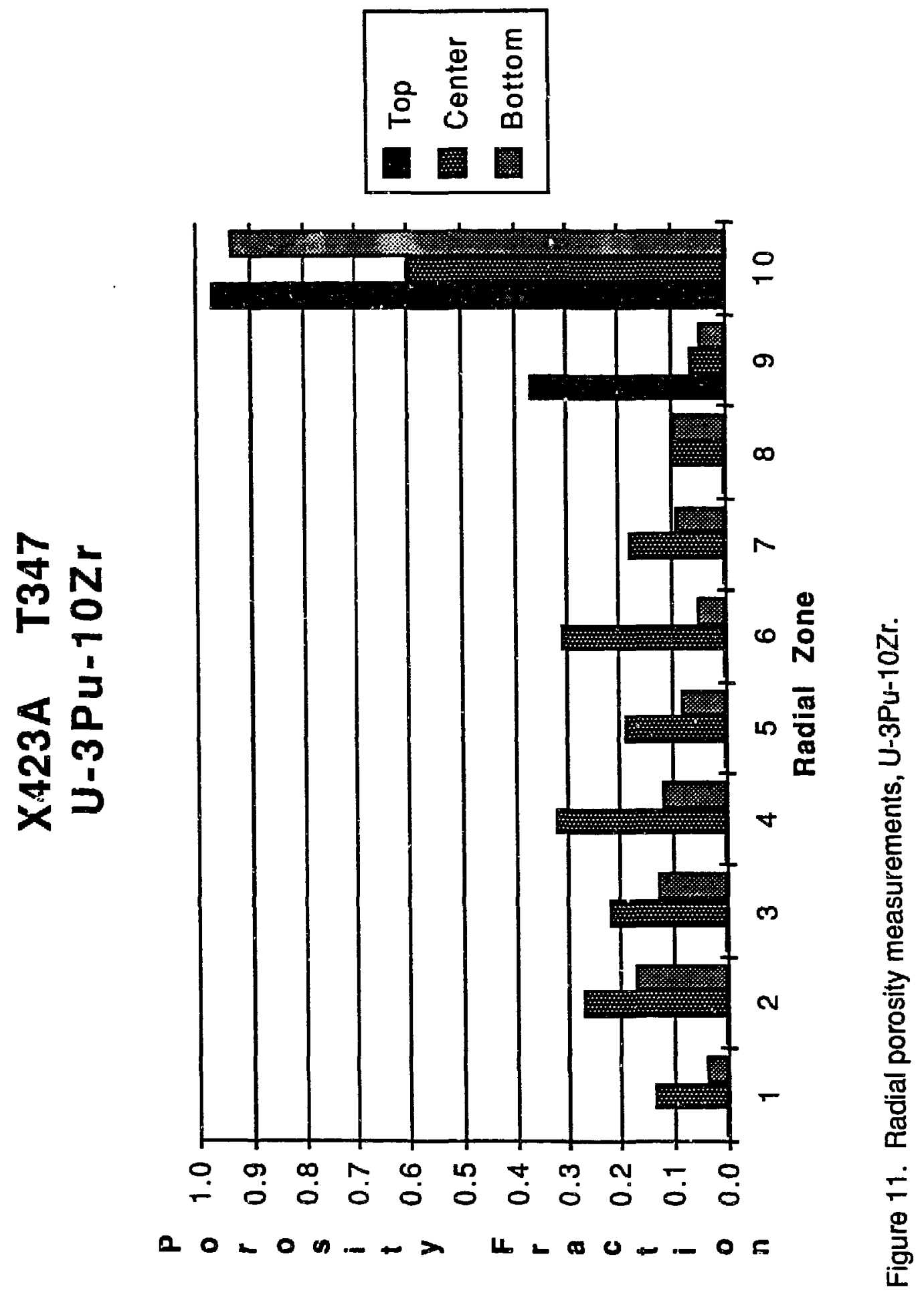




\section{$\begin{array}{ll}X 23 A & T 347\end{array}$ $\mathrm{U}-3 \mathrm{Pu}-10 \mathrm{Zr}$}

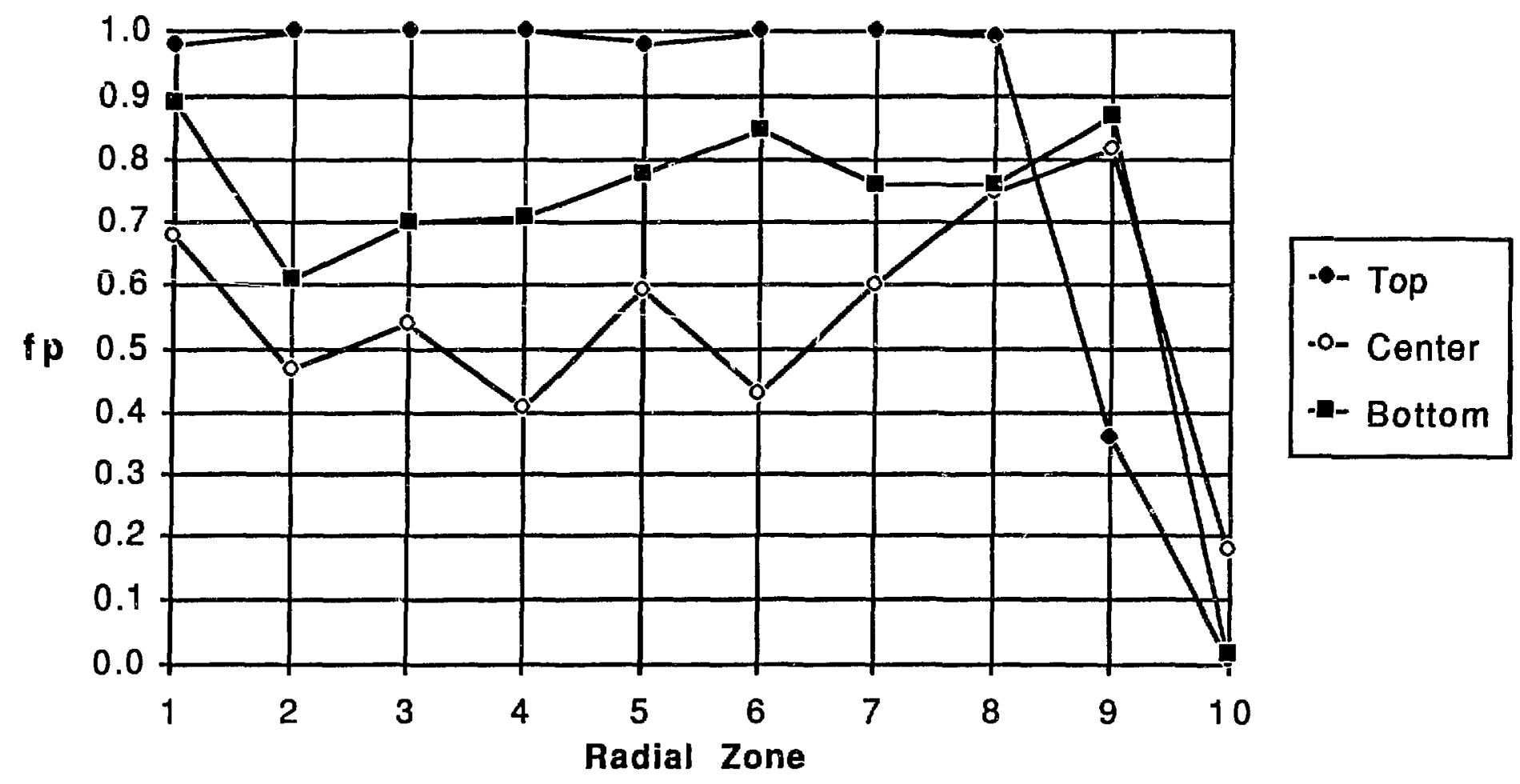

Figure 12. Multiplier for thermal conductivity redurtion, U-3Pu-10Zr. 


\section{X423A T347 $\mathrm{U}-3 \mathrm{Pu}-10 \mathrm{Zr}$}

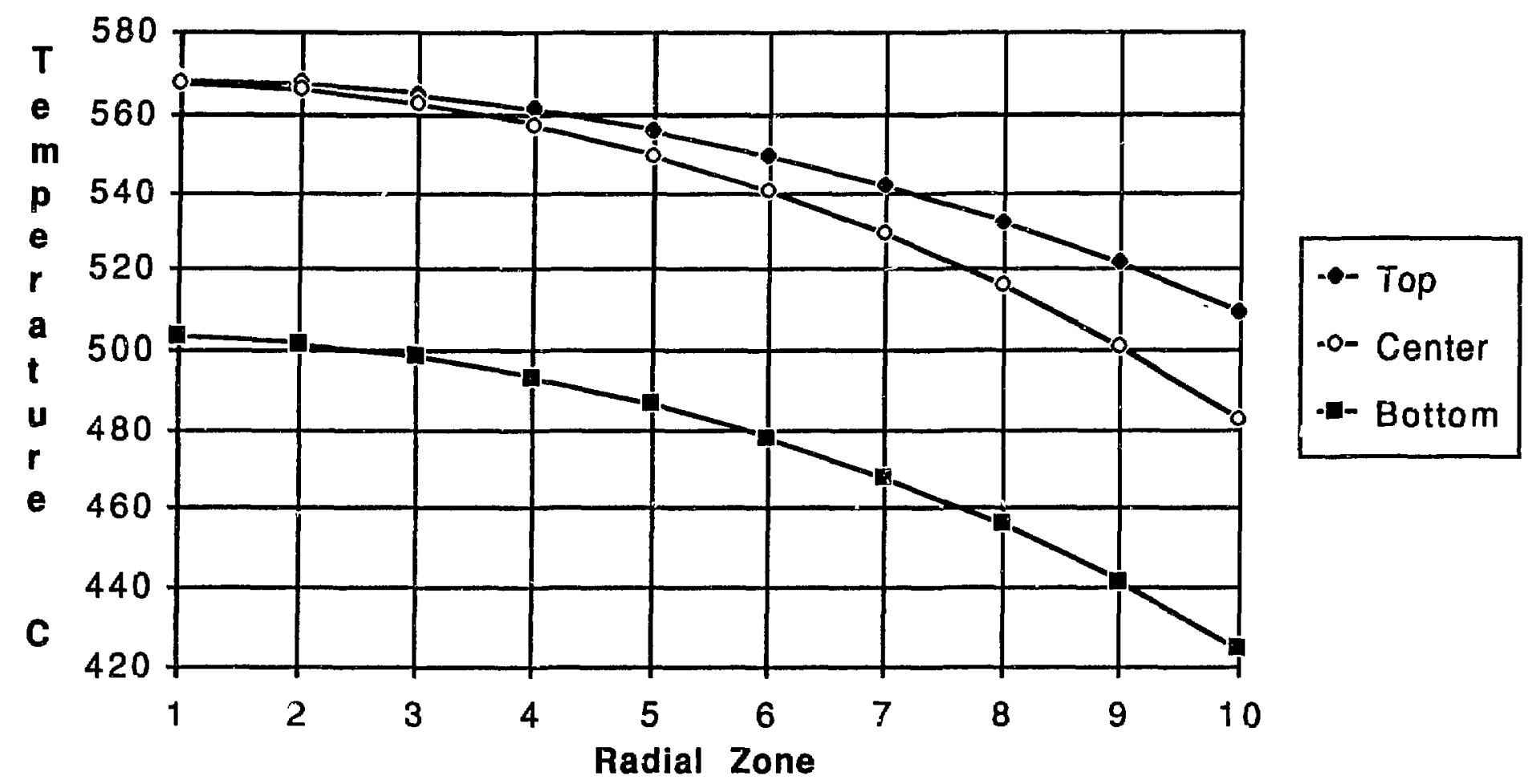

Figure 13. Radial temperature profiles in fresh fuel model, U-3Pu-10Zr. 


\section{X423A T347 U-3Pu-10Zr}

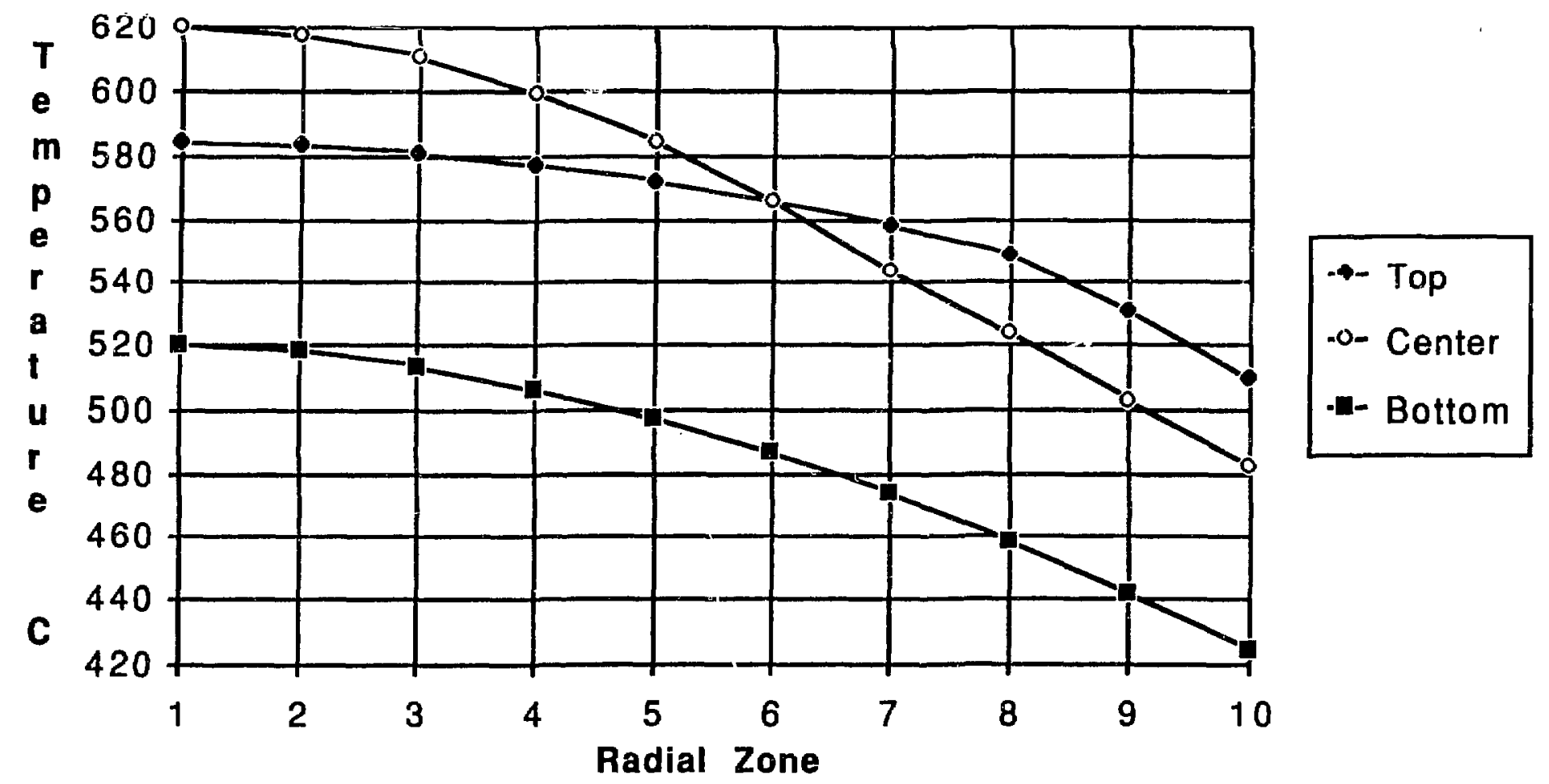

Figure 14. Radial temperature profiles after reducing thermal conductivity in zones 1-9, U-3Pu-10Zr. 


\section{$\begin{array}{ll}X 423 A & T 347\end{array}$ \\ U-3Pu-10Zr}

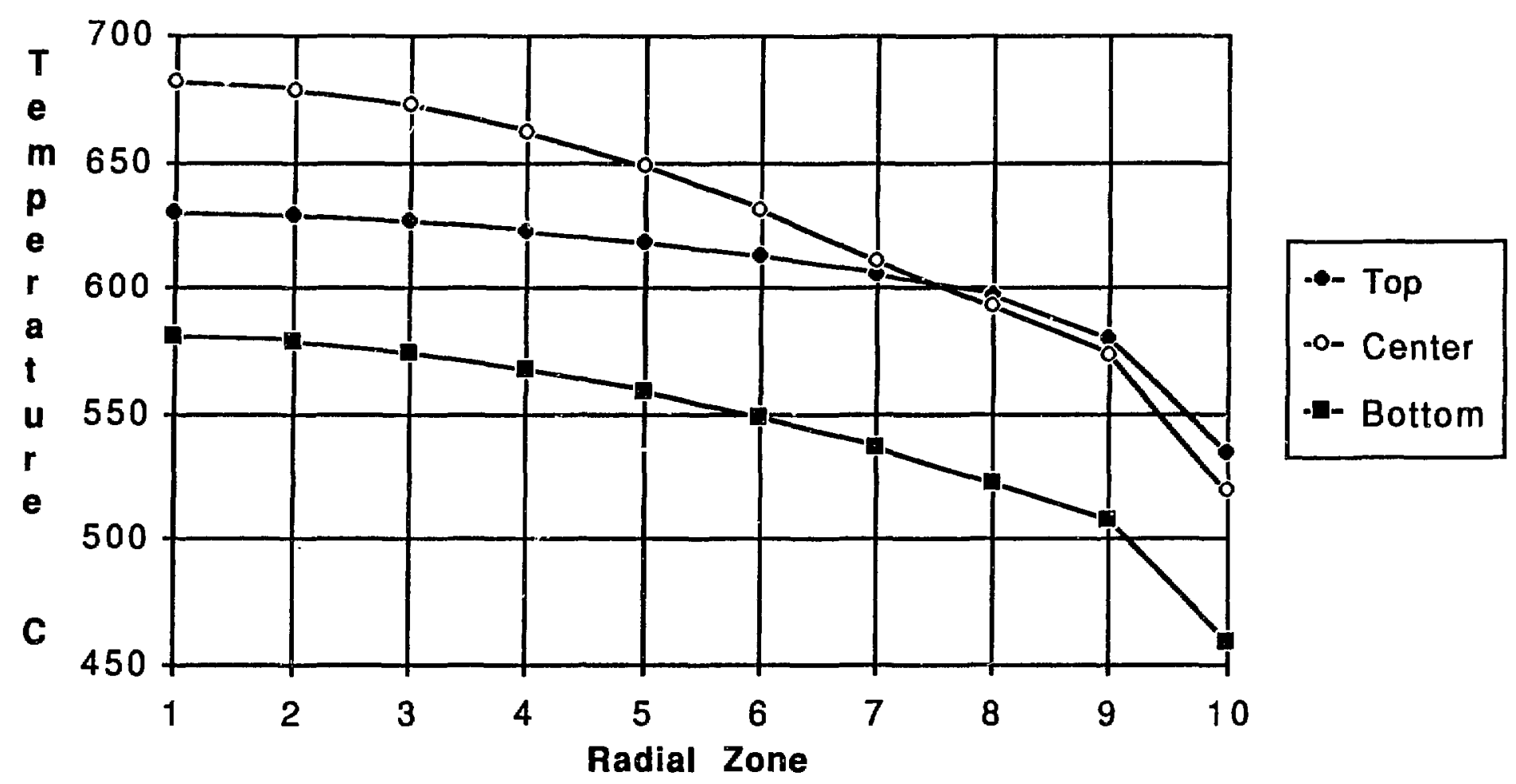

Figure 15. Radial temperature profiles with reduced thermal conductivity in all zones, U-3Pu-10Zr. 


\section{X423A T358 \\ $\mathrm{U}-8 \mathrm{Pu}-10 \mathrm{Zr}$}

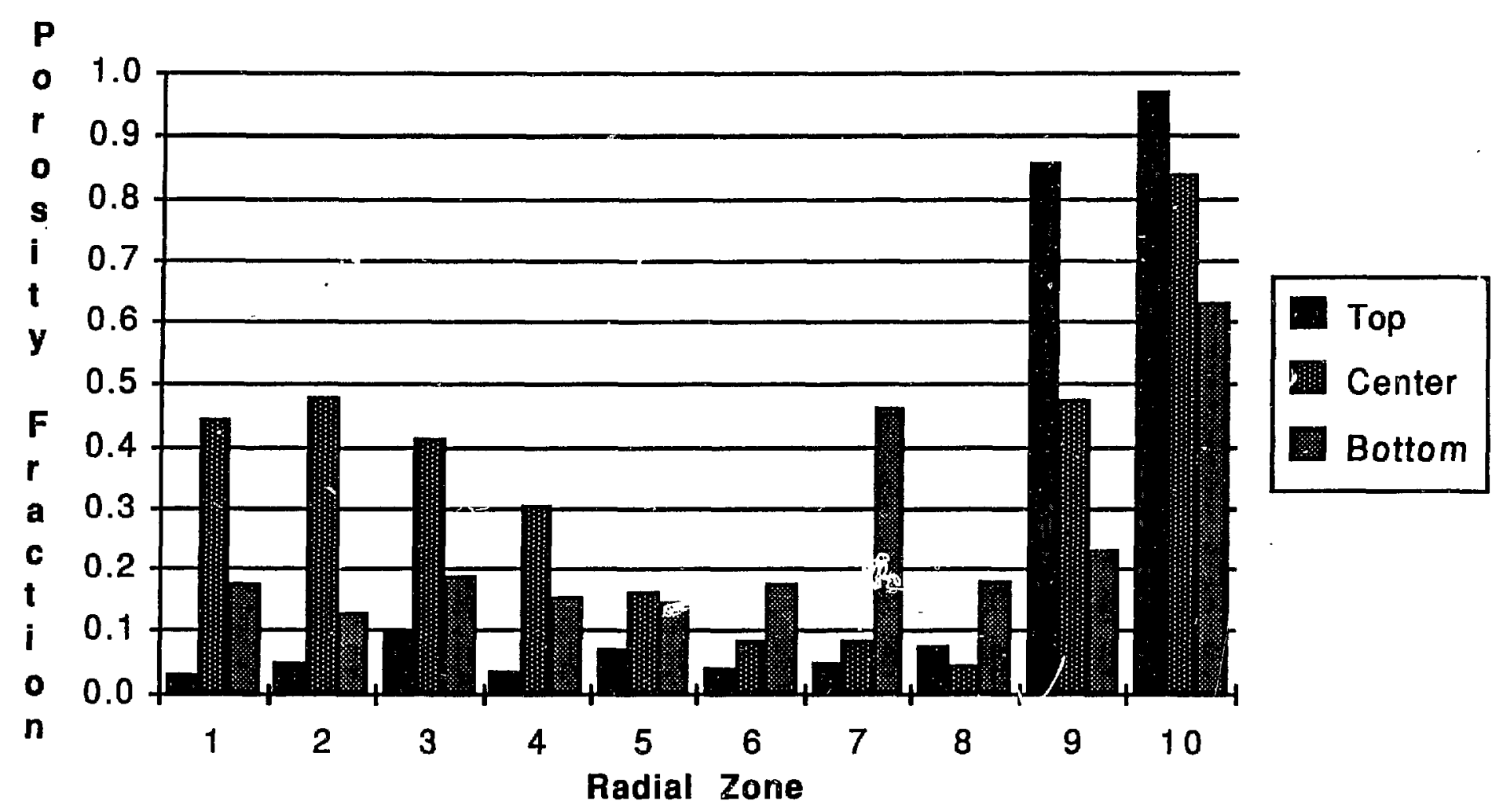

Figure 16. Radial porosity measurements, $\mathrm{U}-8 \mathrm{Pu}-10 \mathrm{Zr}$. 


\section{X423A T358 \\ $\mathrm{U}-8 \mathrm{Pu}-10 \mathrm{Zr}$}

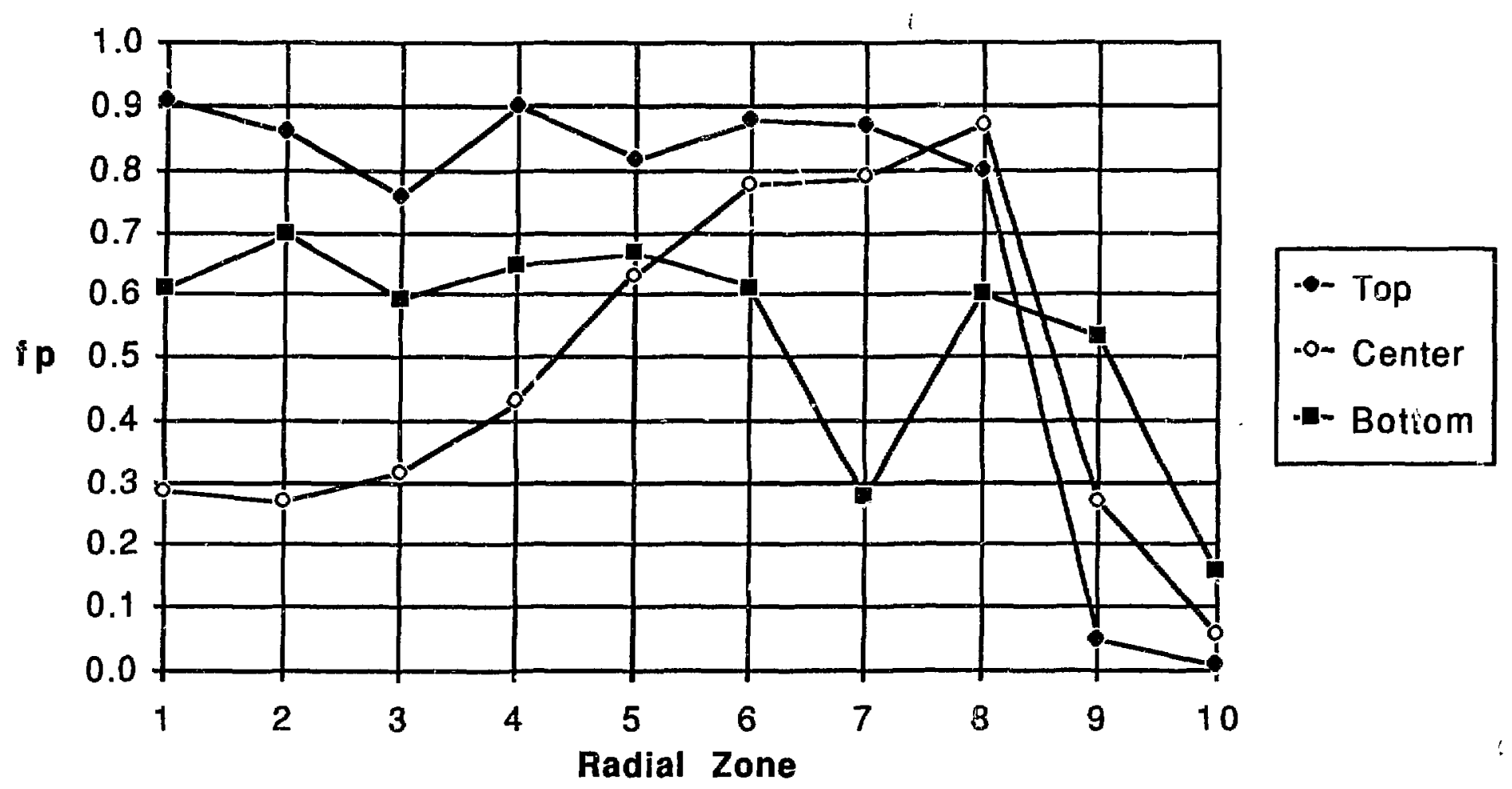

Figure 17. Multiplier for thermal conductivity reduction, U-8Pu-10Zr. 


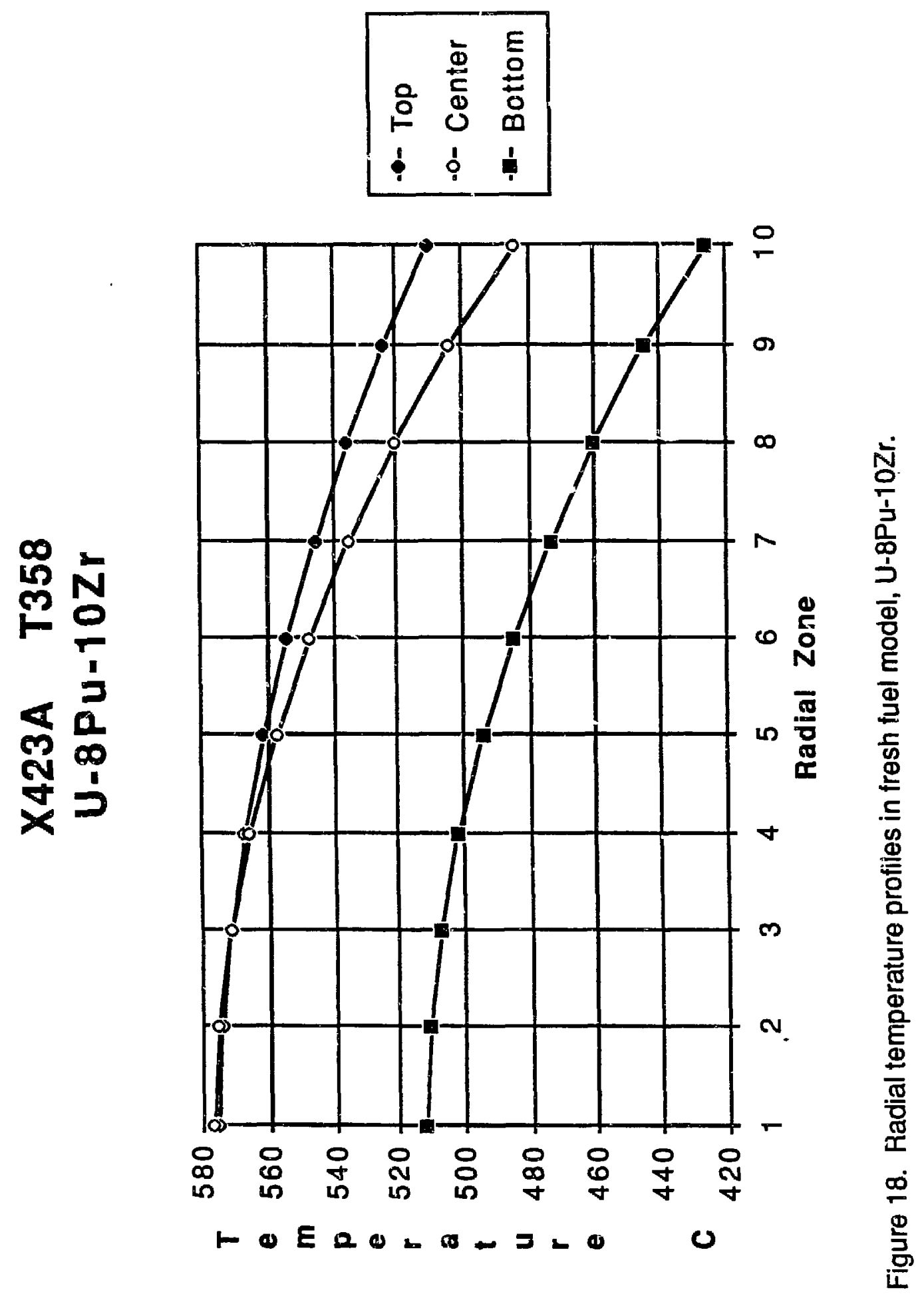




\section{X423A T358 \\ $\mathrm{U}-8 \mathrm{Pu}-10 \mathrm{Zr}$}

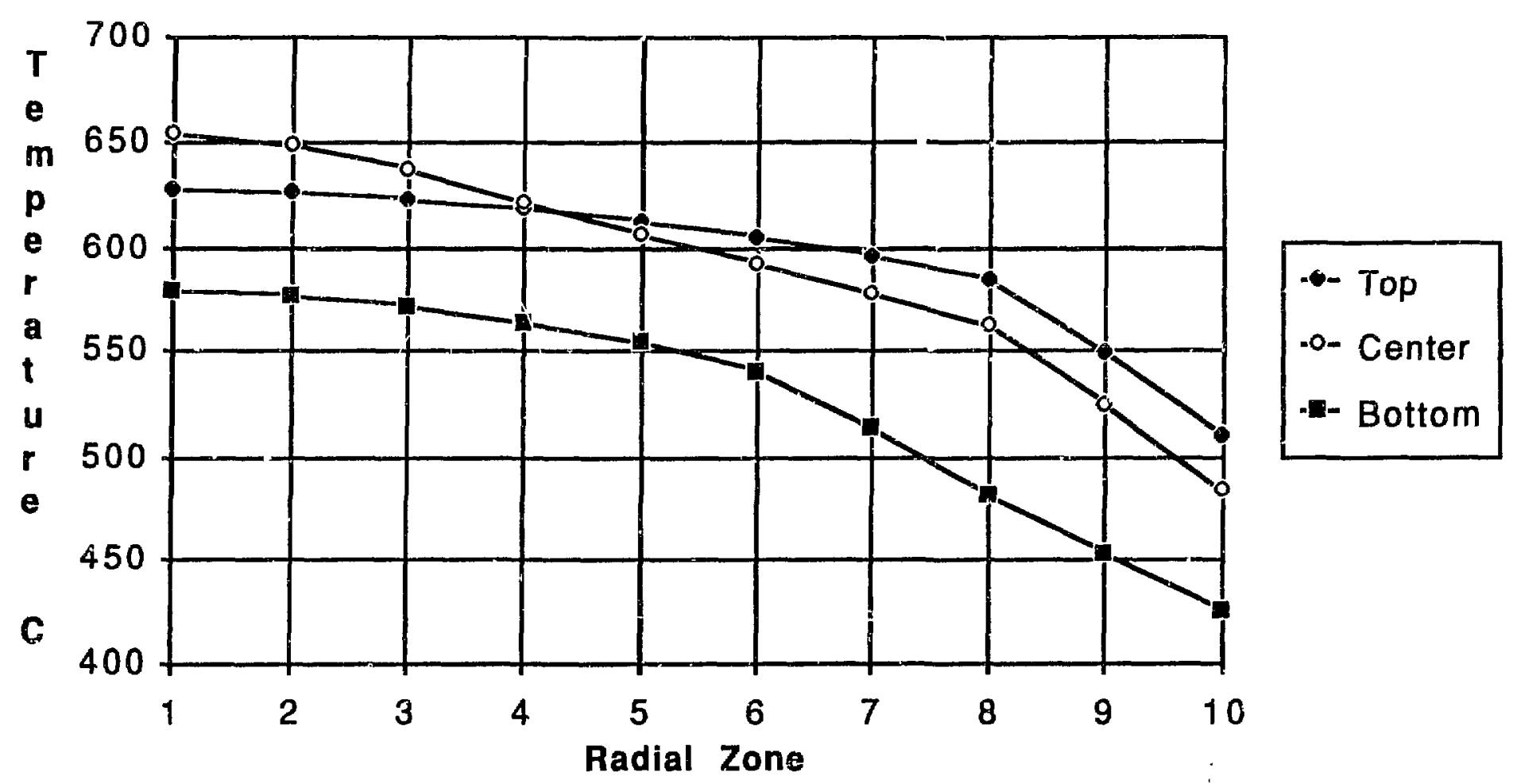

Figure 19. Radial temperatures profiles after rediucing thermal conductivity in zones $1-9, \mathrm{U}-\mathrm{BPu}-10 \mathrm{Zr}$. 


\section{X423A T358 $\mathrm{U}-8 \mathrm{Pu}-10 \mathrm{Zr}$}

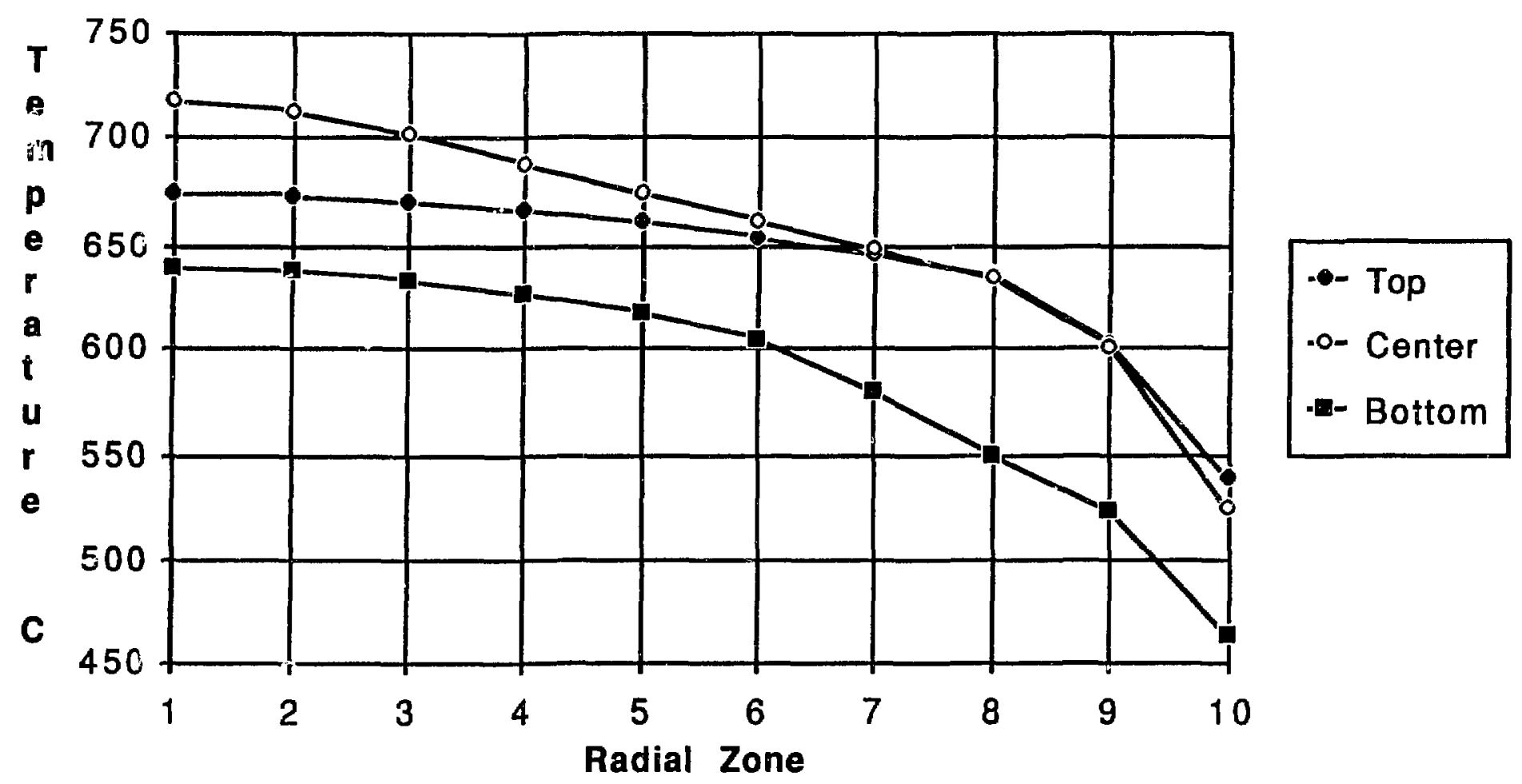

Figure 20. Radial temperature profiles with reduced thermal conductivity in all zones, U-8Pu-10Zr. 
X423A T305

$\mathrm{U}-19 \mathrm{Pu}-10 \mathrm{Zr}$

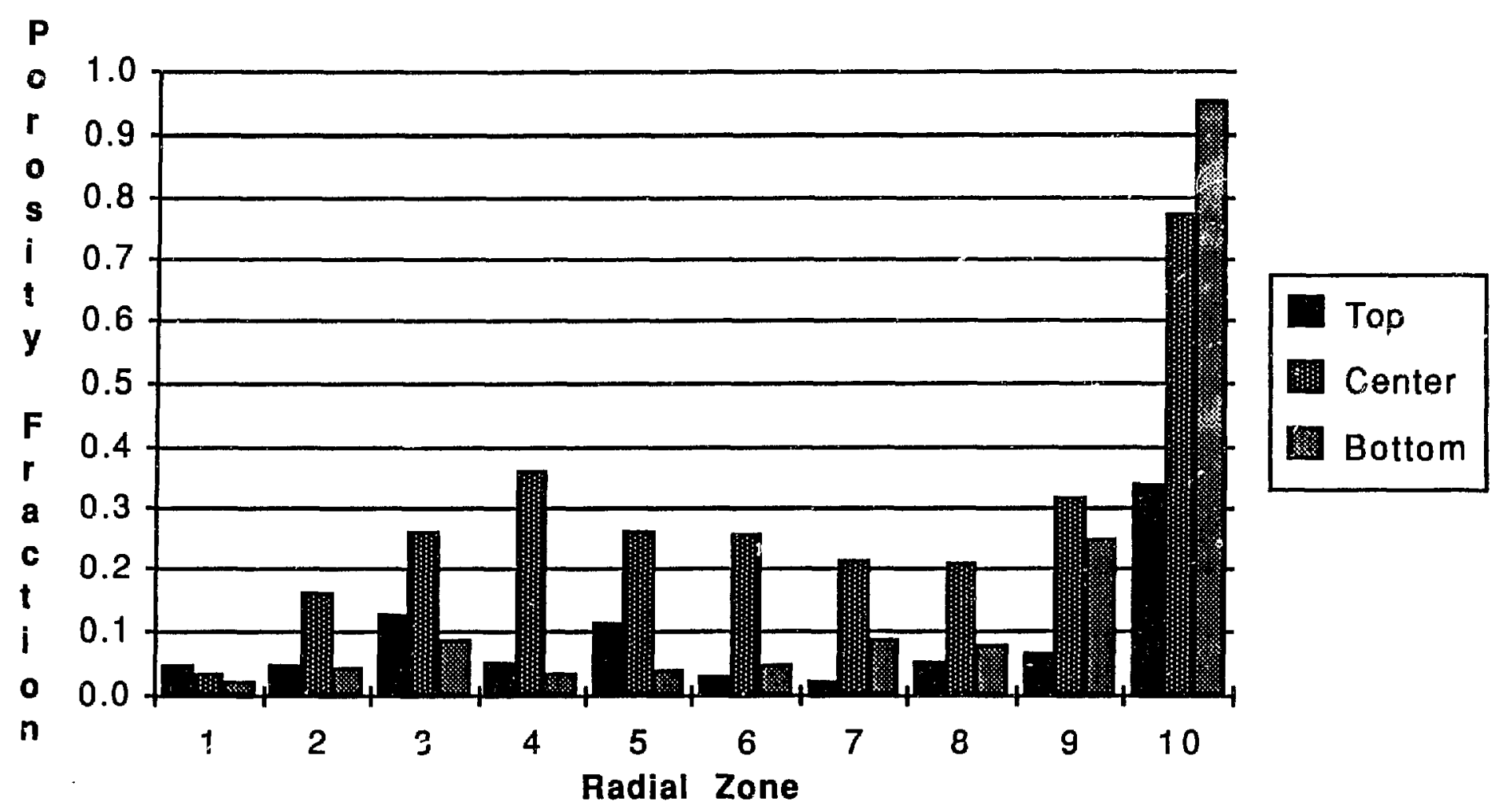

Figure 21. Radial porosity measurements, U-19Pu-10Zr. 


\section{X423.4 T305 $\mathrm{U}-19 \mathrm{Pu}-10 \mathrm{Zr}$}

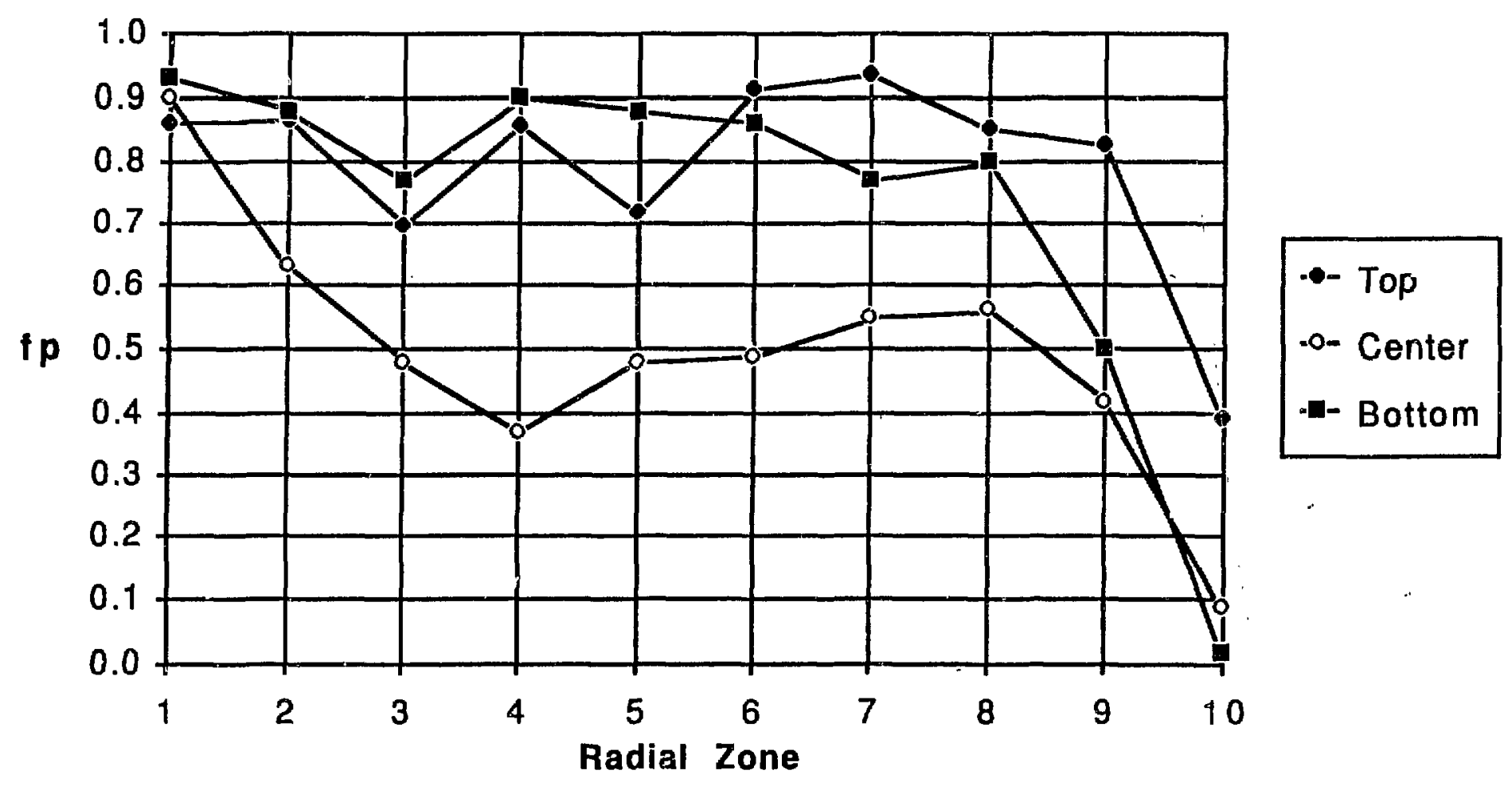

Figure 22. Multiplier for thermal conductivity reduction, U-19Pu-10Zr. 


\section{X423A T305 \\ $U-19 P u=10 Z r$}

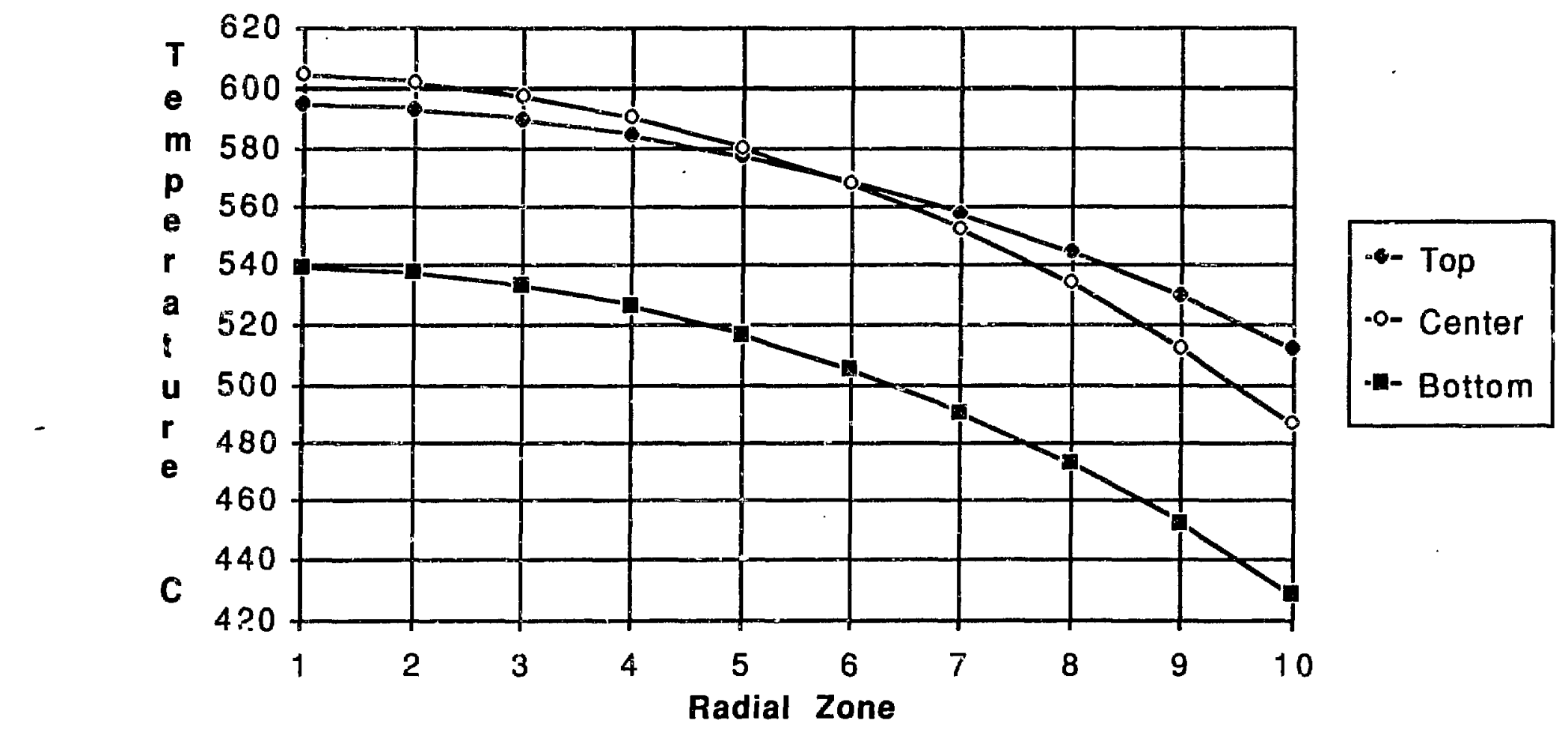

Figure 23. Radial temperature profiles in fresh fuel model, U-19Pu-10Zr. 


\section{X423A T305 $\mathrm{U}-19 \mathrm{Pu}-10 \mathrm{Zr}$}

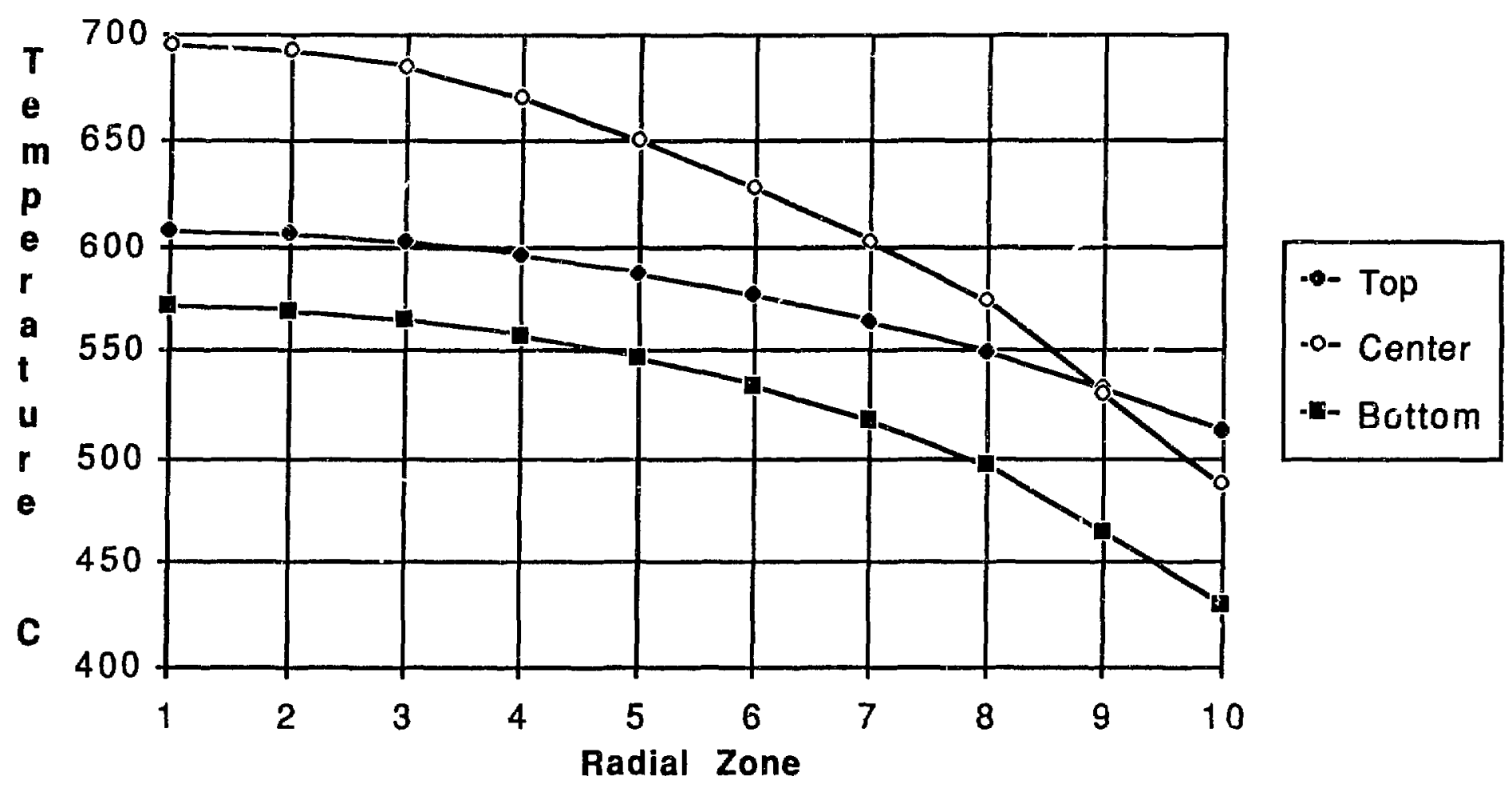

Figure 24. Radial temperature profiles after reducing thermal conductivity in zones $1-9, \mathrm{U}-19 \mathrm{Pu}-10 \mathrm{Zr}$. 


\section{X423A T305}

\section{U-19Pu-10Zr}

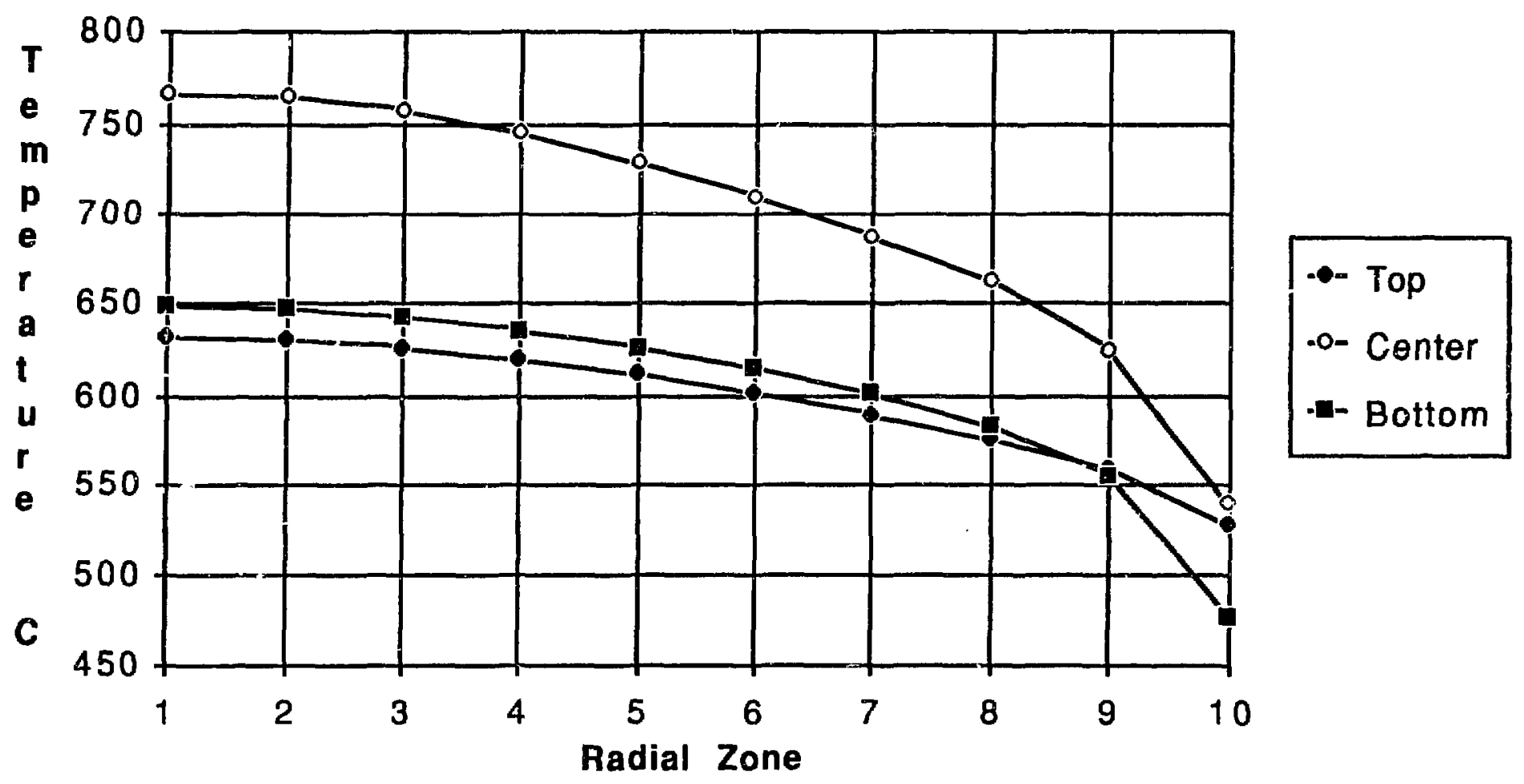

Figure 25. Radial temperature profiles with reduced thermal conductivity in all zones, U-19Pu-10Zr. 


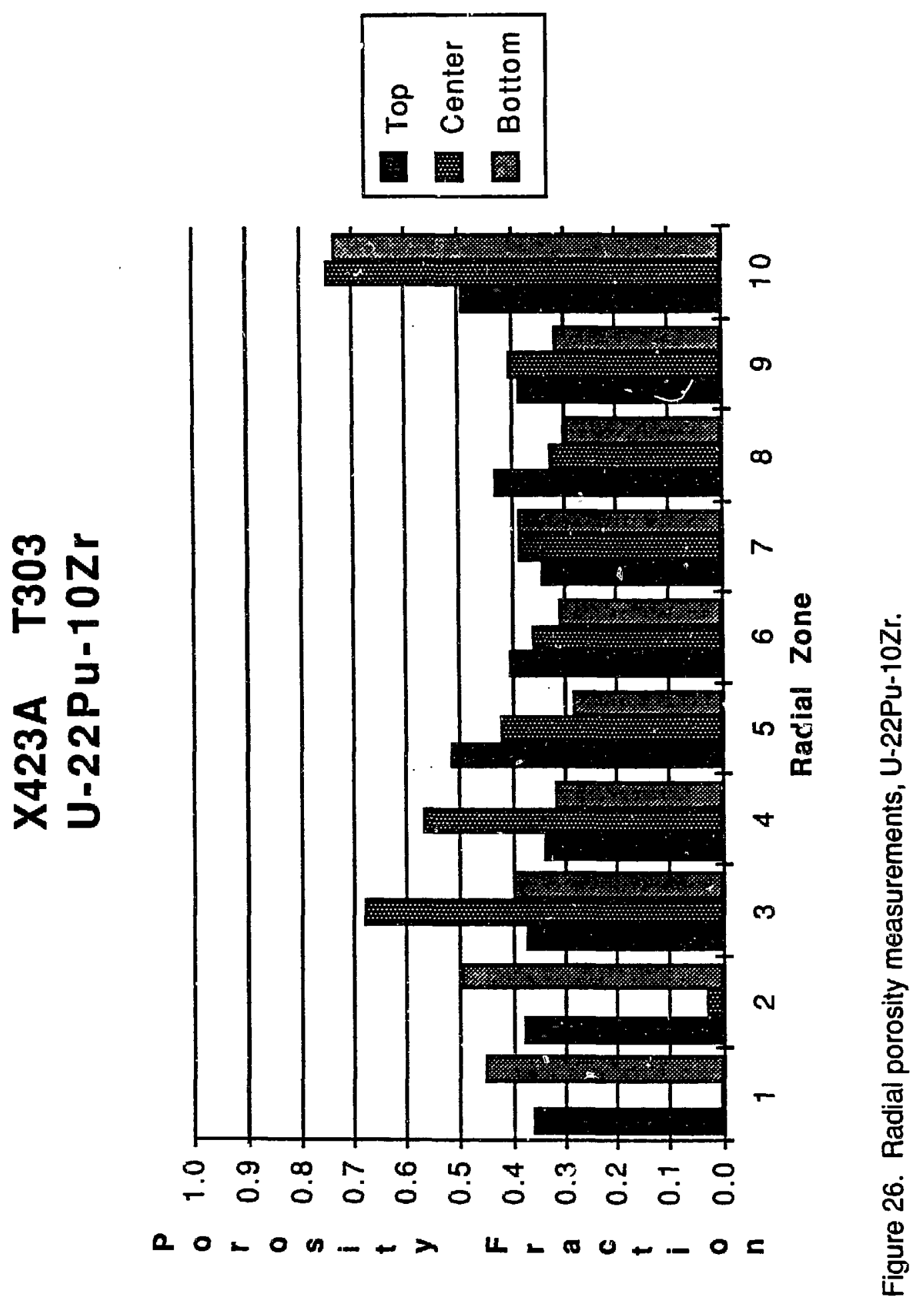




\section{$X 423 A \quad T 303$ $\mathrm{U}-22 \mathrm{Pu}-10 \mathrm{Zr}$}

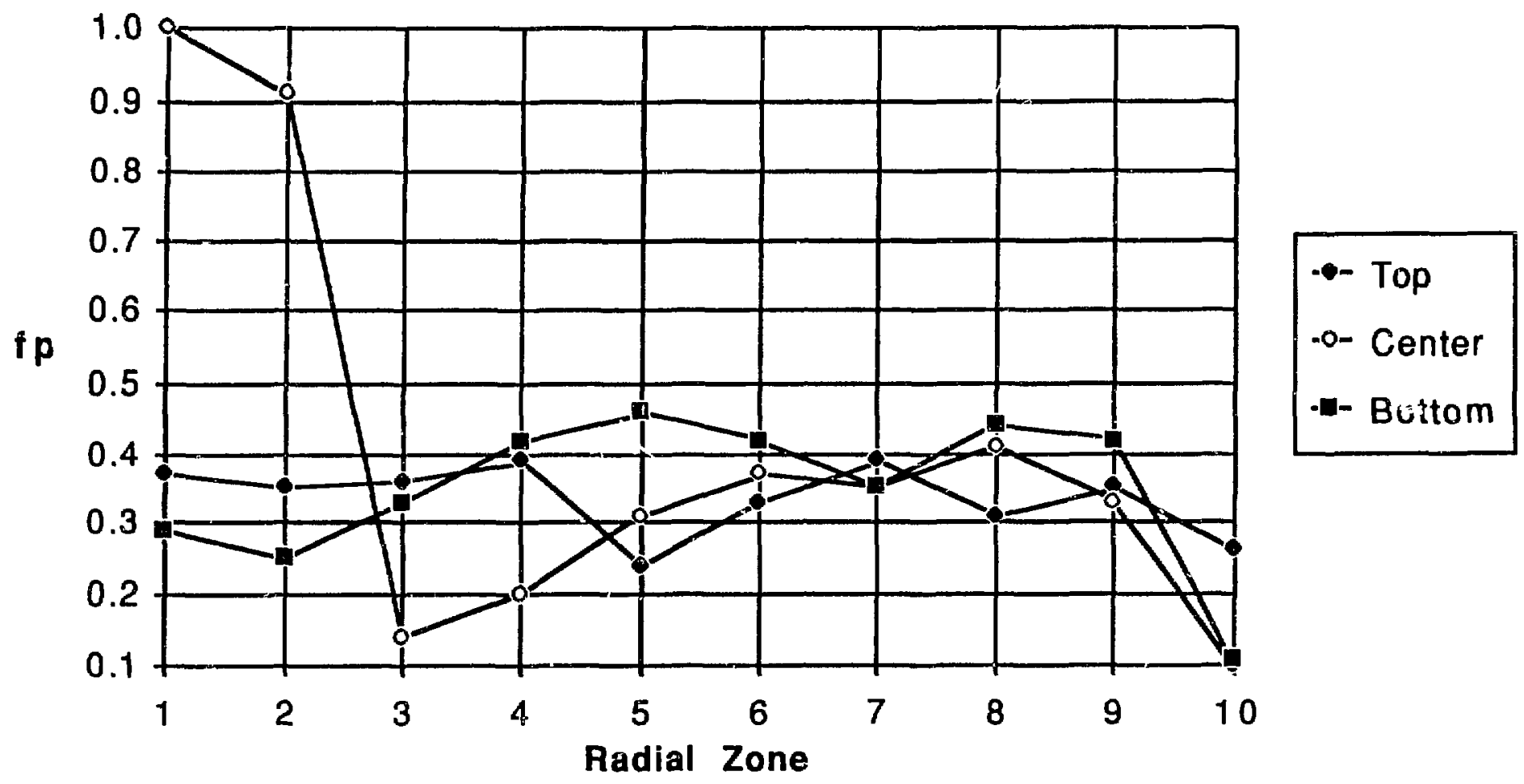

Figure 27. Multiplier for thermal conductivity reduction, U-22Pu-10Zr. 


\section{X423A T303 \\ $\mathrm{U}-22 \mathrm{Pu}-10 \mathrm{Zr}$}

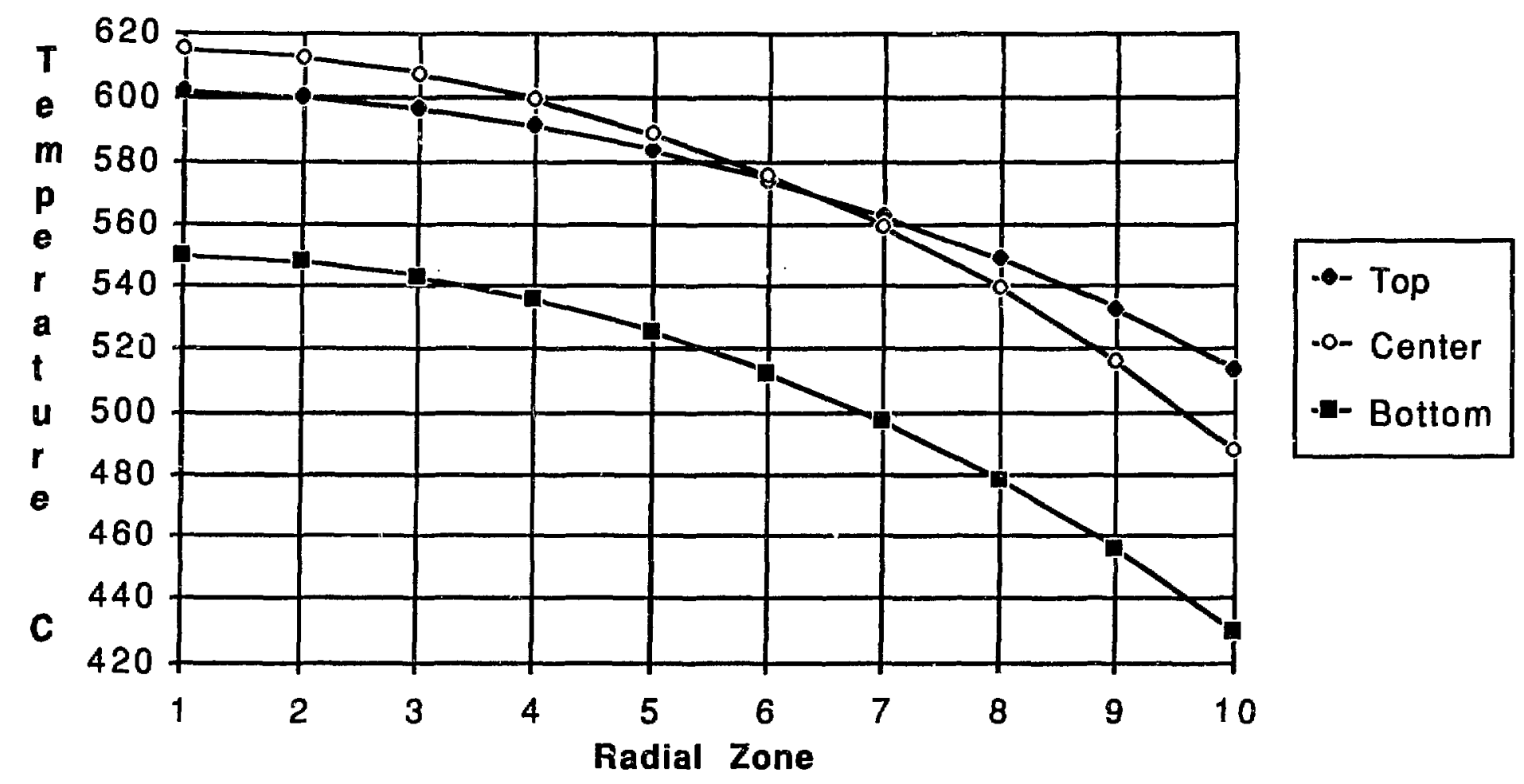

Figure 28. Radial temperature profiles in fresh fuel model, U-22Pu-10Zr. 


\section{X423A T303 \\ $\mathrm{U}-22 \mathrm{Pu}-10 \mathrm{Zr}$}

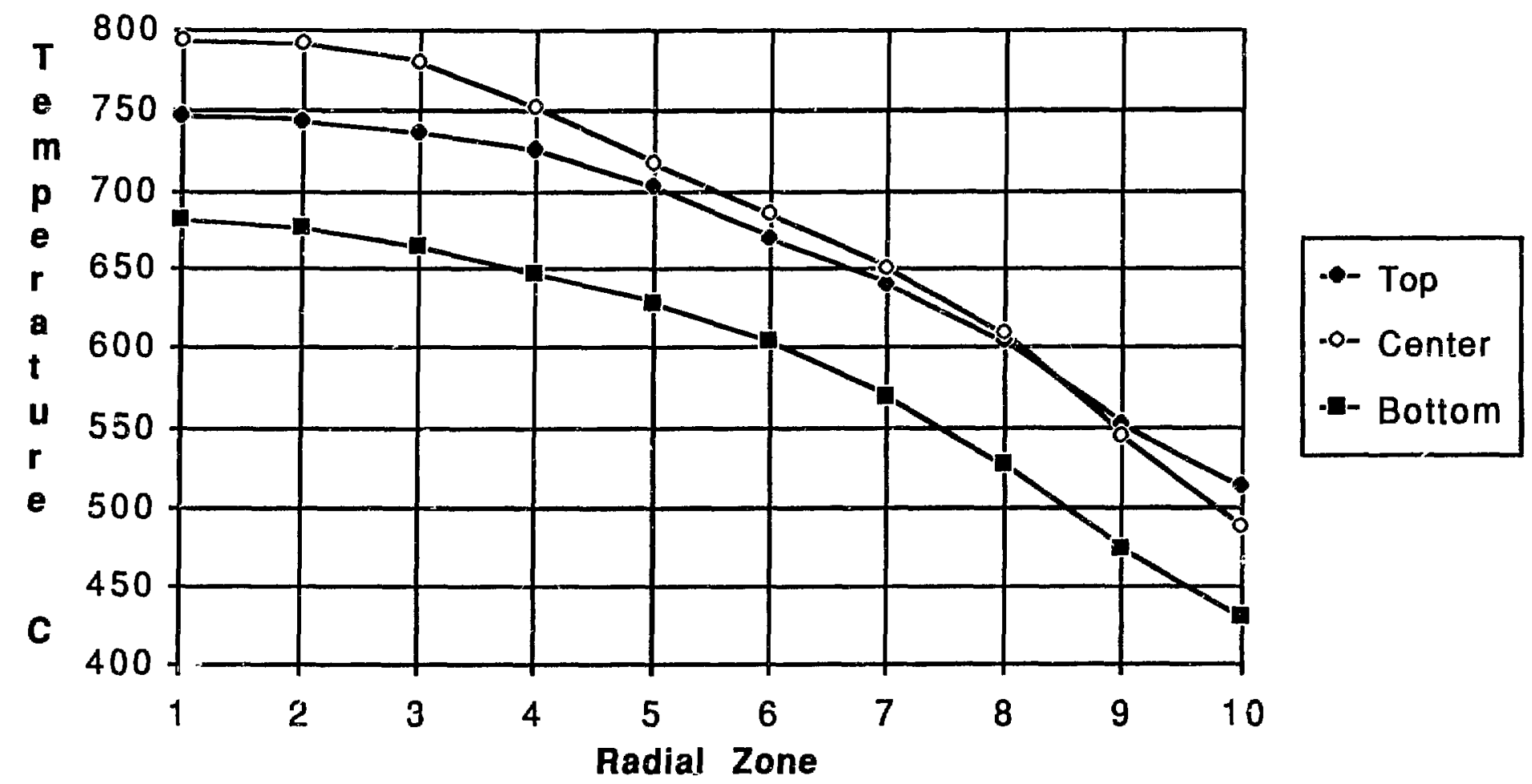

Figure 29. Radial temperature profiles after reducing thermal conductivity in zones 1-9, U-22Pu-10Zr. 


\section{X423A T303 $\mathrm{U}-22 \mathrm{Pu}-10 \mathrm{Zr}$}

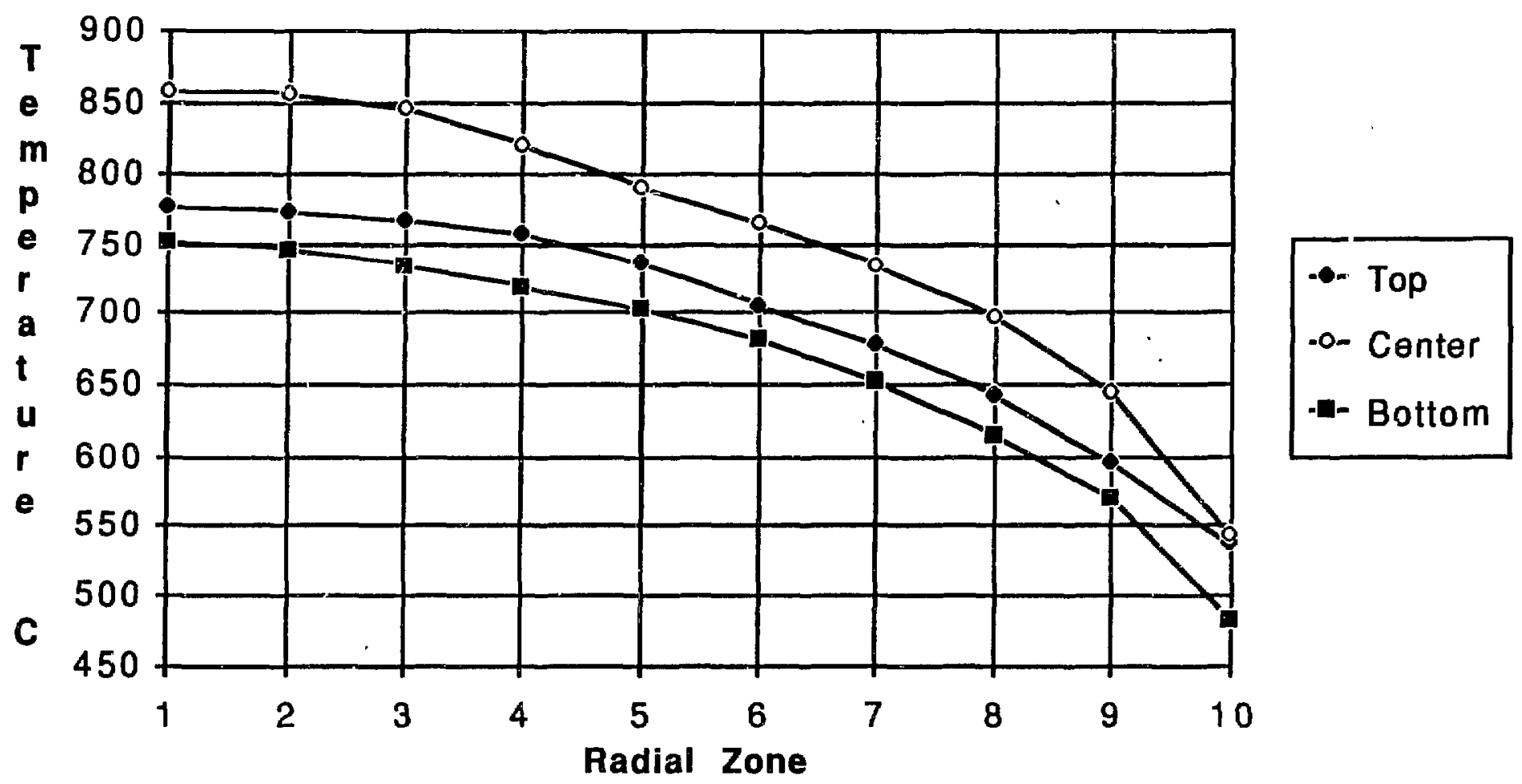

Figure 30. Radial temperature profiles with reduced thermal conductivity in all zones, U-22Pu-10Zr. 


\section{$\mathrm{X} 423 \mathrm{~A} \quad \mathrm{~T} 353$ \\ $\mathrm{U}-26 \mathrm{Pu}-10 \mathrm{Zr}$}

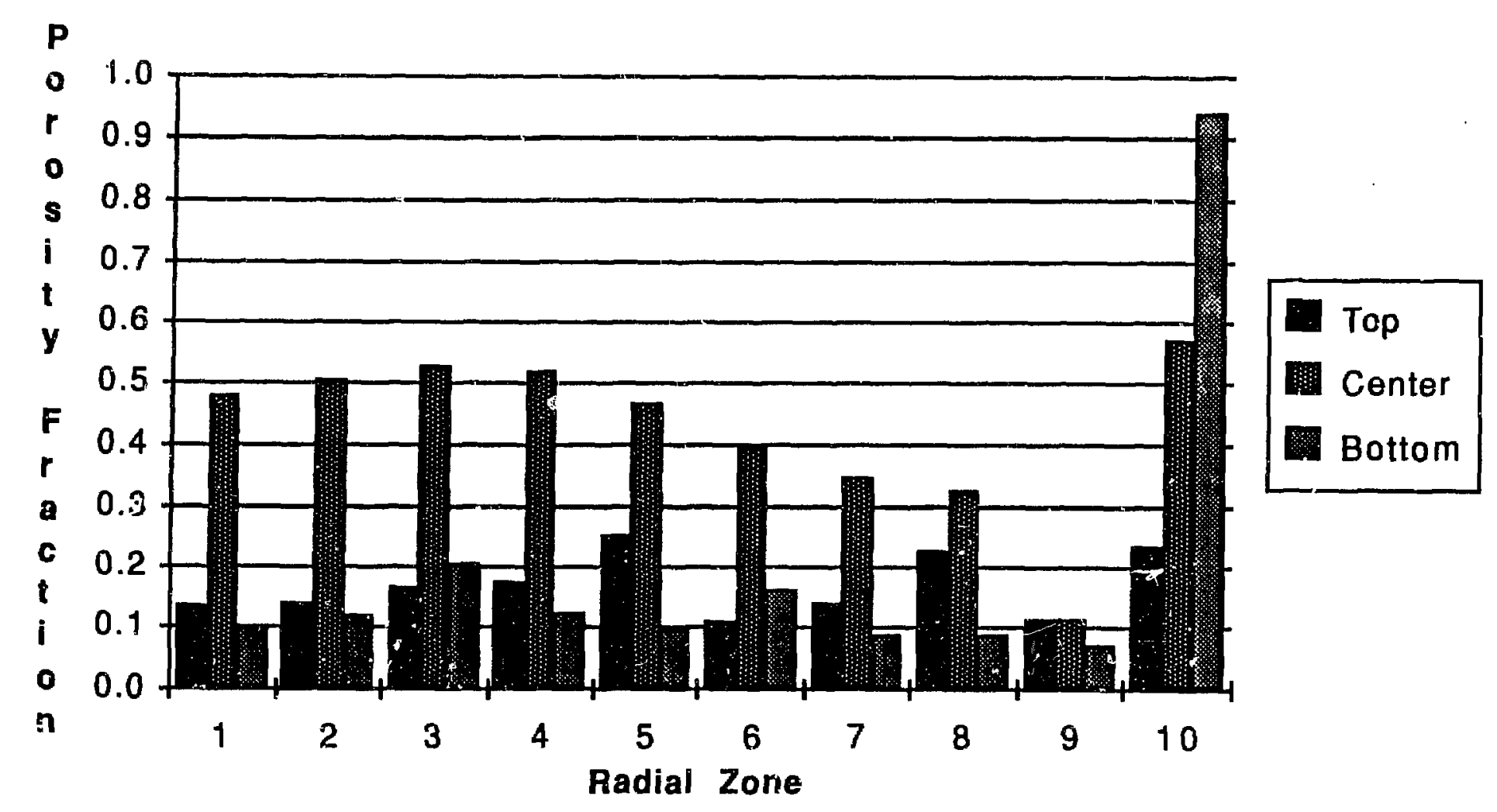

Figure 3i. Radial porosity measurements, U-26Pu-10Zr. 


\section{$X 423 A \quad T 353$ \\ $U-26 P u-10 Z r$}

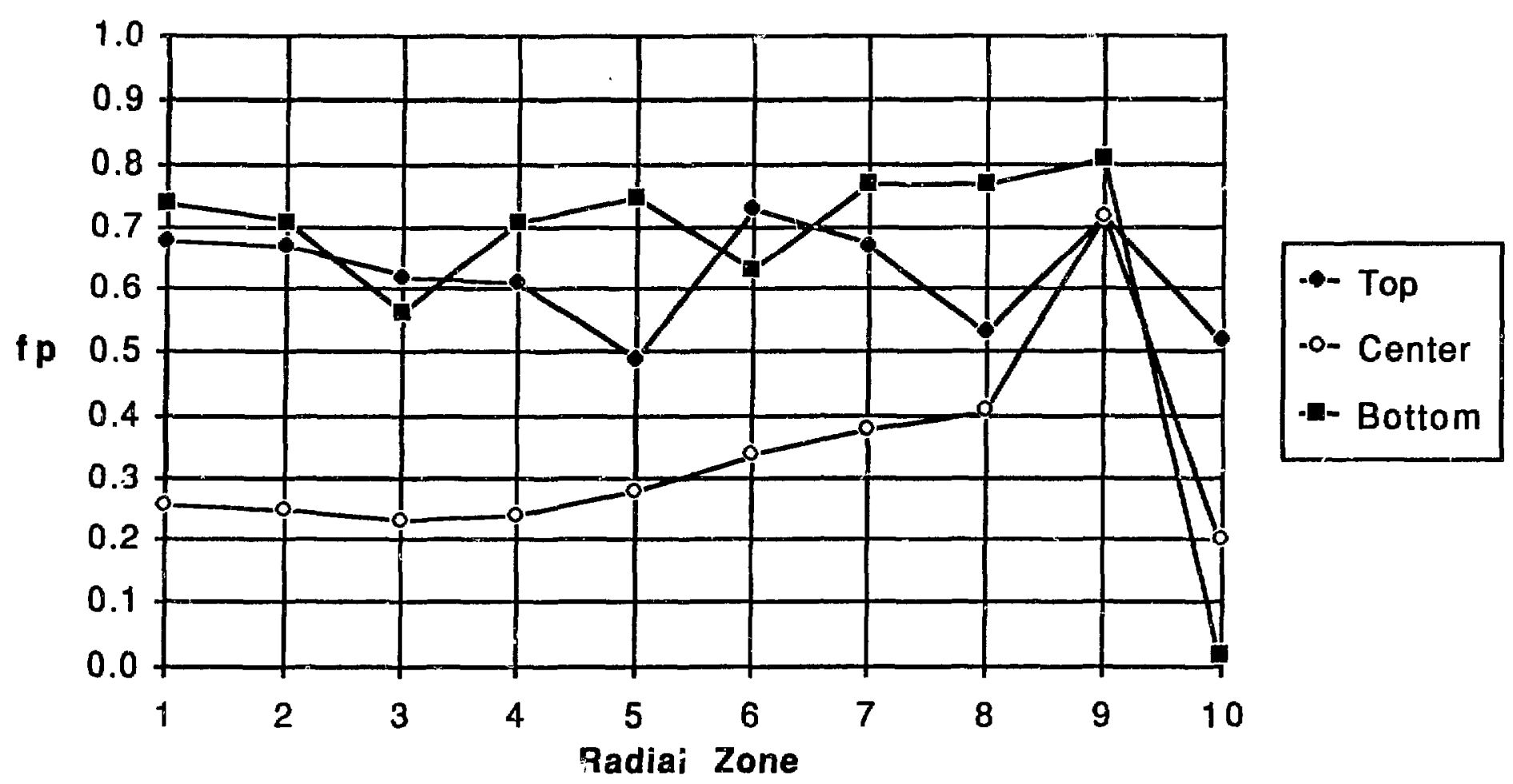

Figure 32. Multiplier for thermal conductivity reduction, U-26Pu-10Zr. 


\section{$\mathrm{X} 423 \mathrm{~A} \quad \mathrm{~T} 353$ $\mathrm{U}-26 \mathrm{Pu}-10 \mathrm{Zr}$}

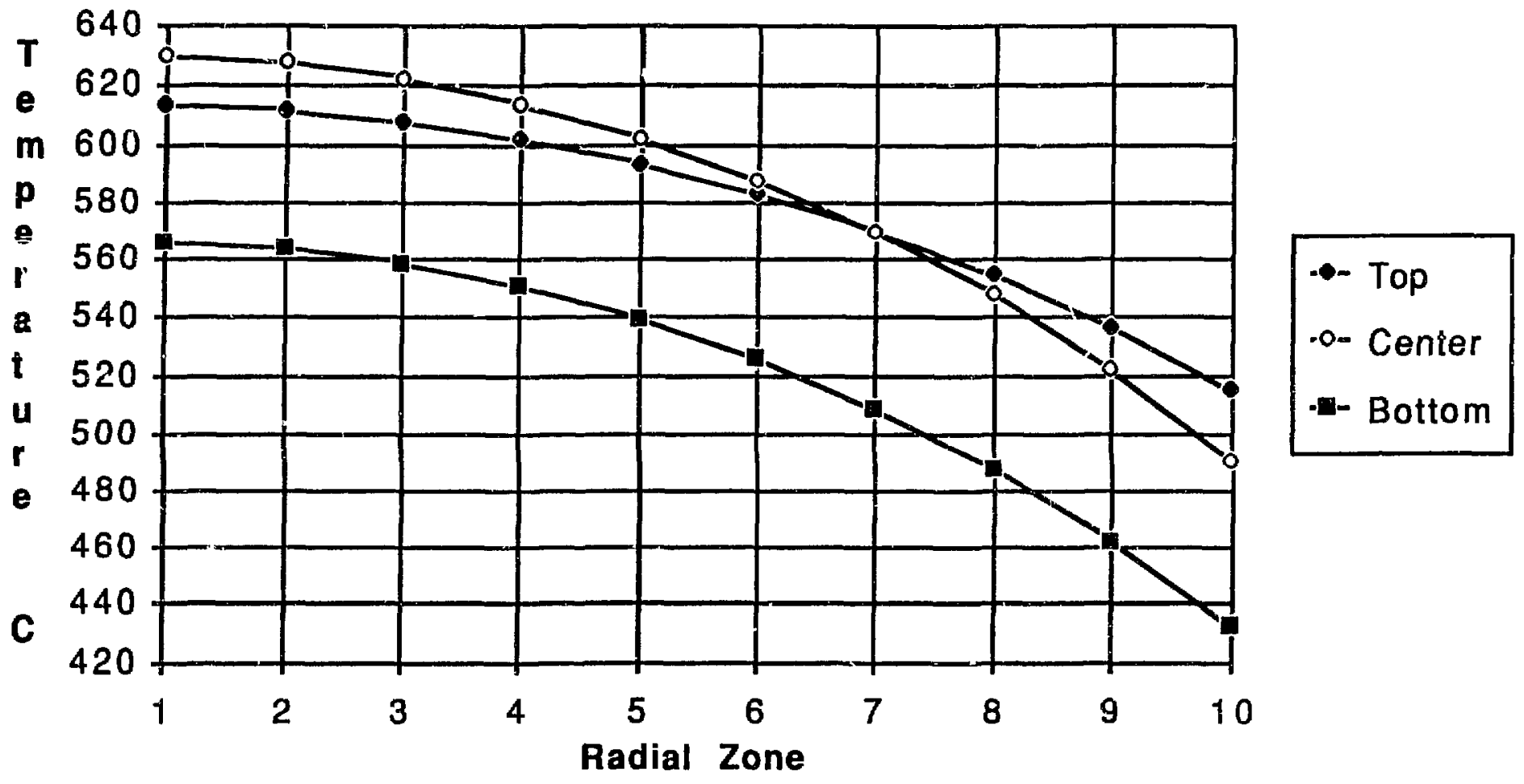

Figure 33. Radial temperature protiles in fresh fuel mociel, U-26Pu-10Zr. 


\section{$X 423 A \quad T 353$ \\ $\mathrm{U}-26 \mathrm{Pu}-10 \mathrm{Zr}$}

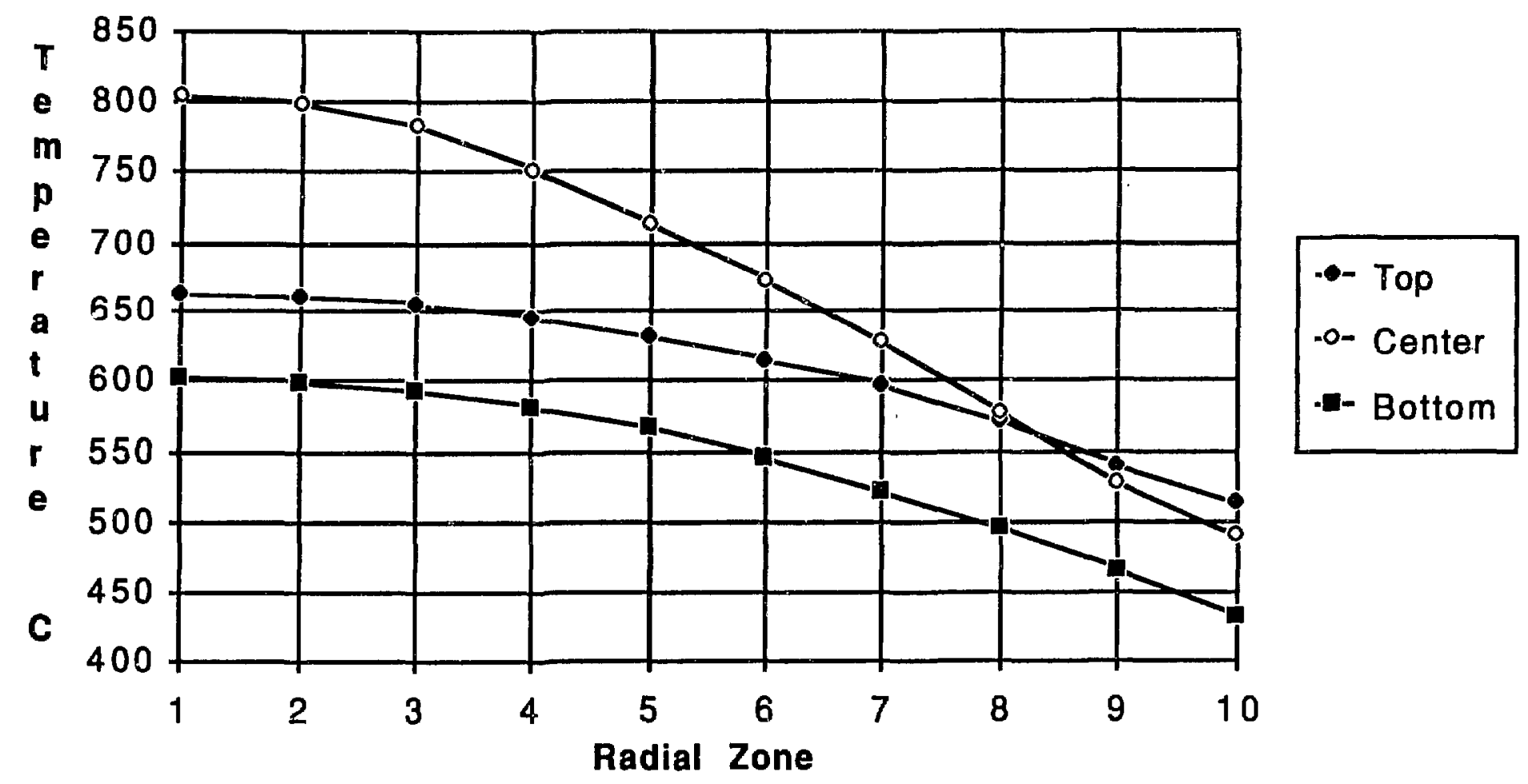

Figure 34. Radial temperature profiles after reducing thermal conductivity in zones 1-9, U-26Pu-10Zr. 


\section{X423A T353 \\ $\mathrm{U}-26 \mathrm{Pu}-10 \mathrm{Zr}$}

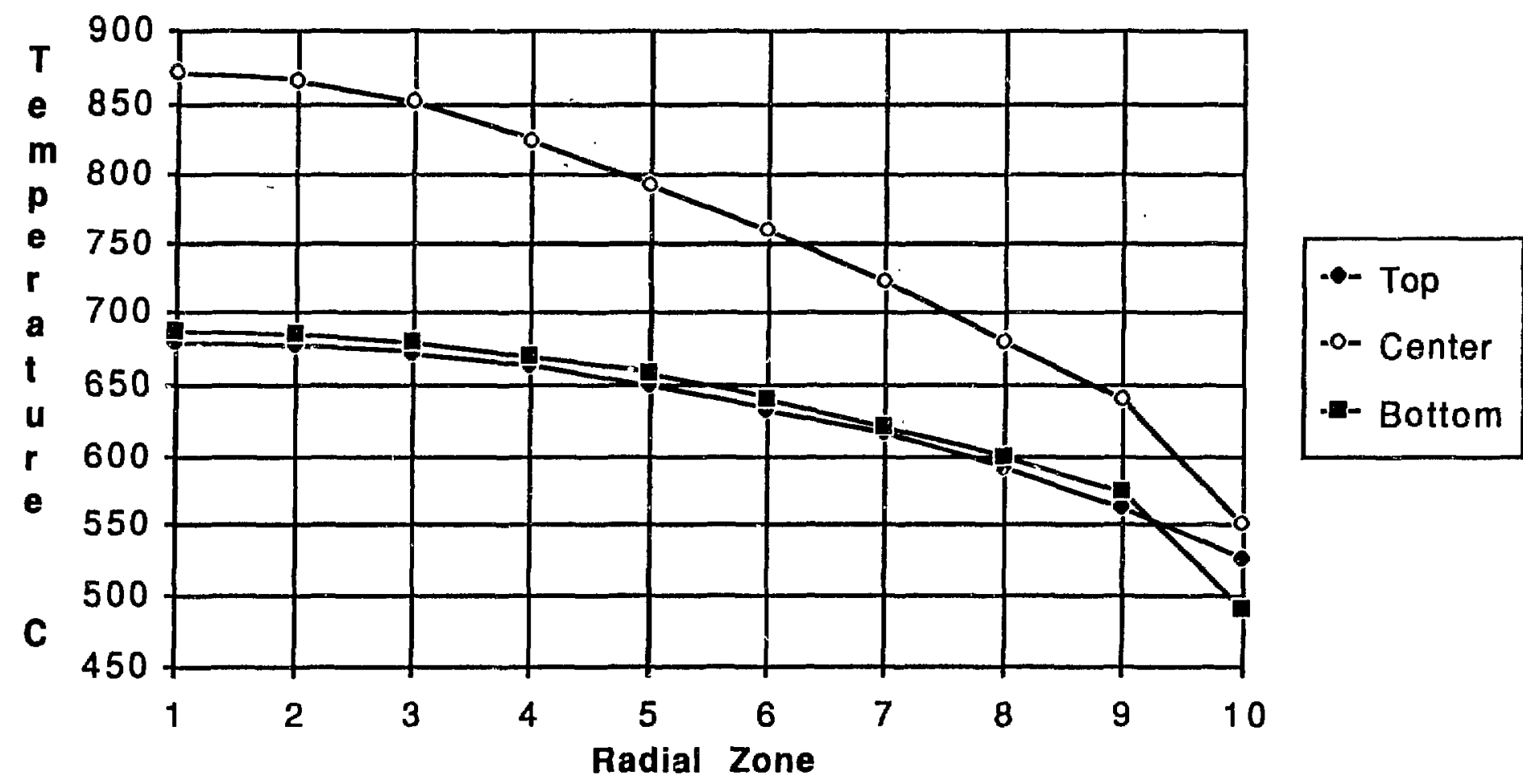

Figure 35. Radial temperature profiles with reduced thermal conductivity in all zones, U-26Pu-10Zr. 

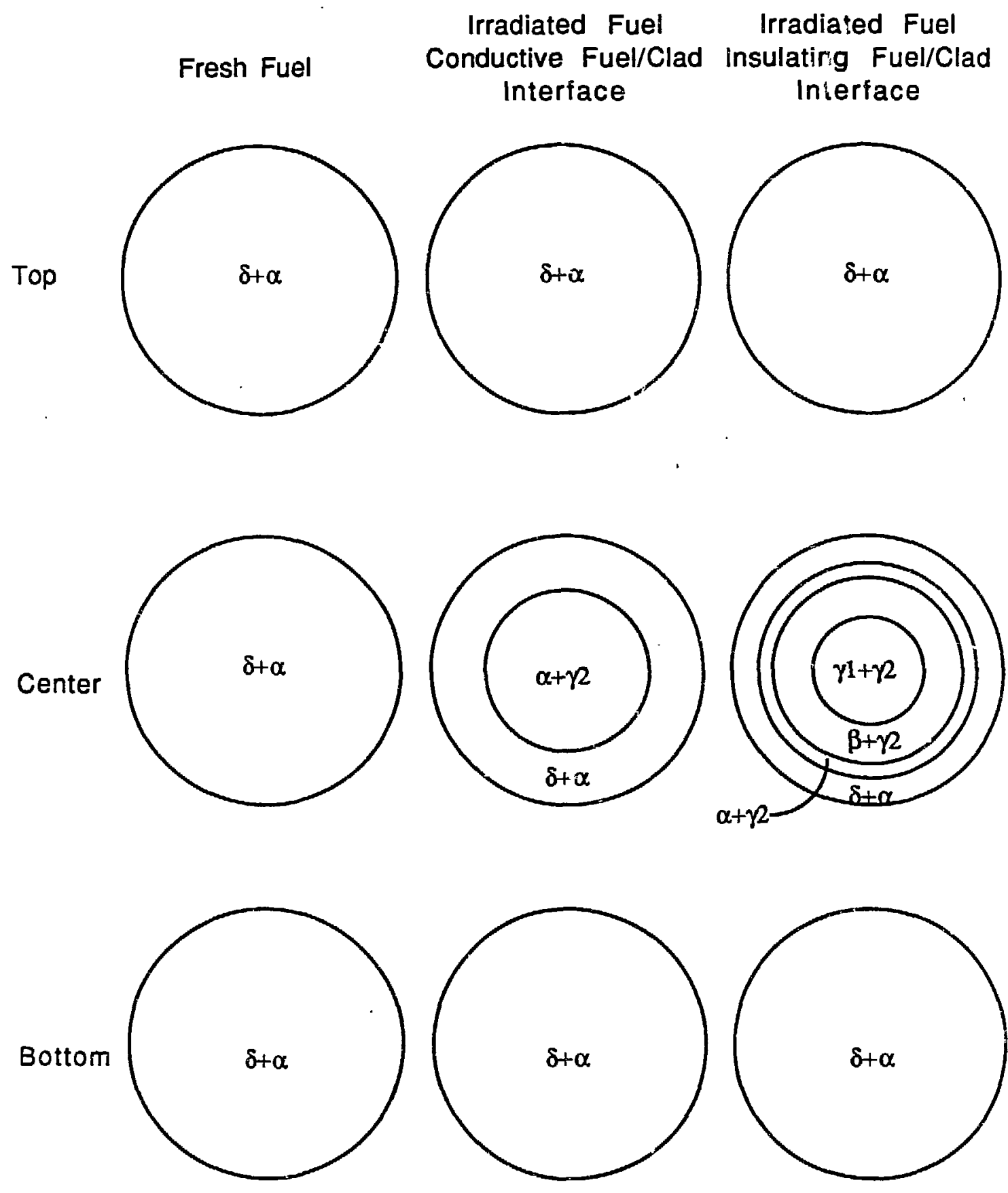

Figure 36. Possible phases and phase boundaries in U-10Zr, element T332. 

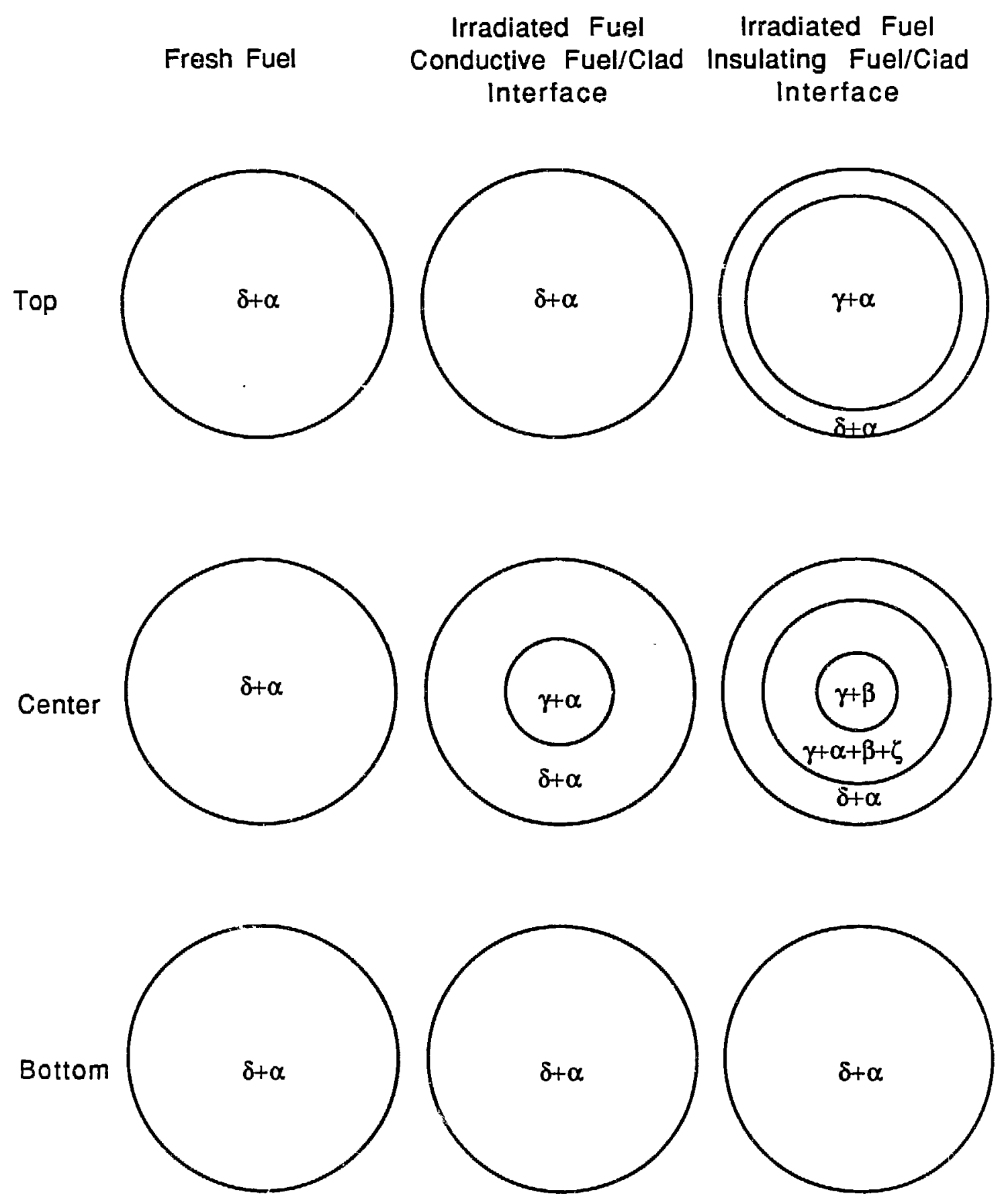

Figure 57 . Possible phases and phase boundaries in li-3Pu-10Zr, element T347. 

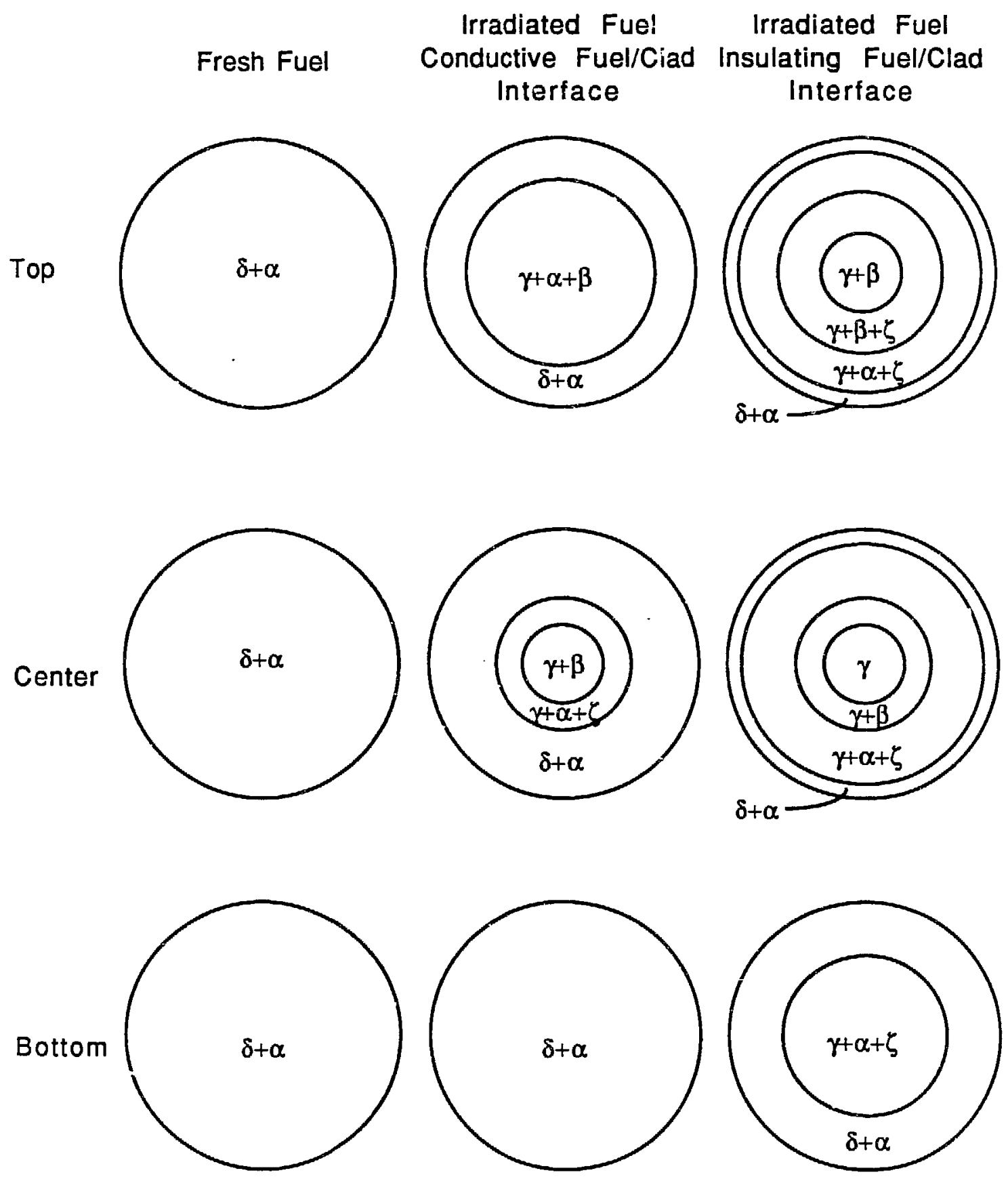

Figure 38. Possible phases and phase boundaries in U-8Pu-10Zr, element $T 358$. 


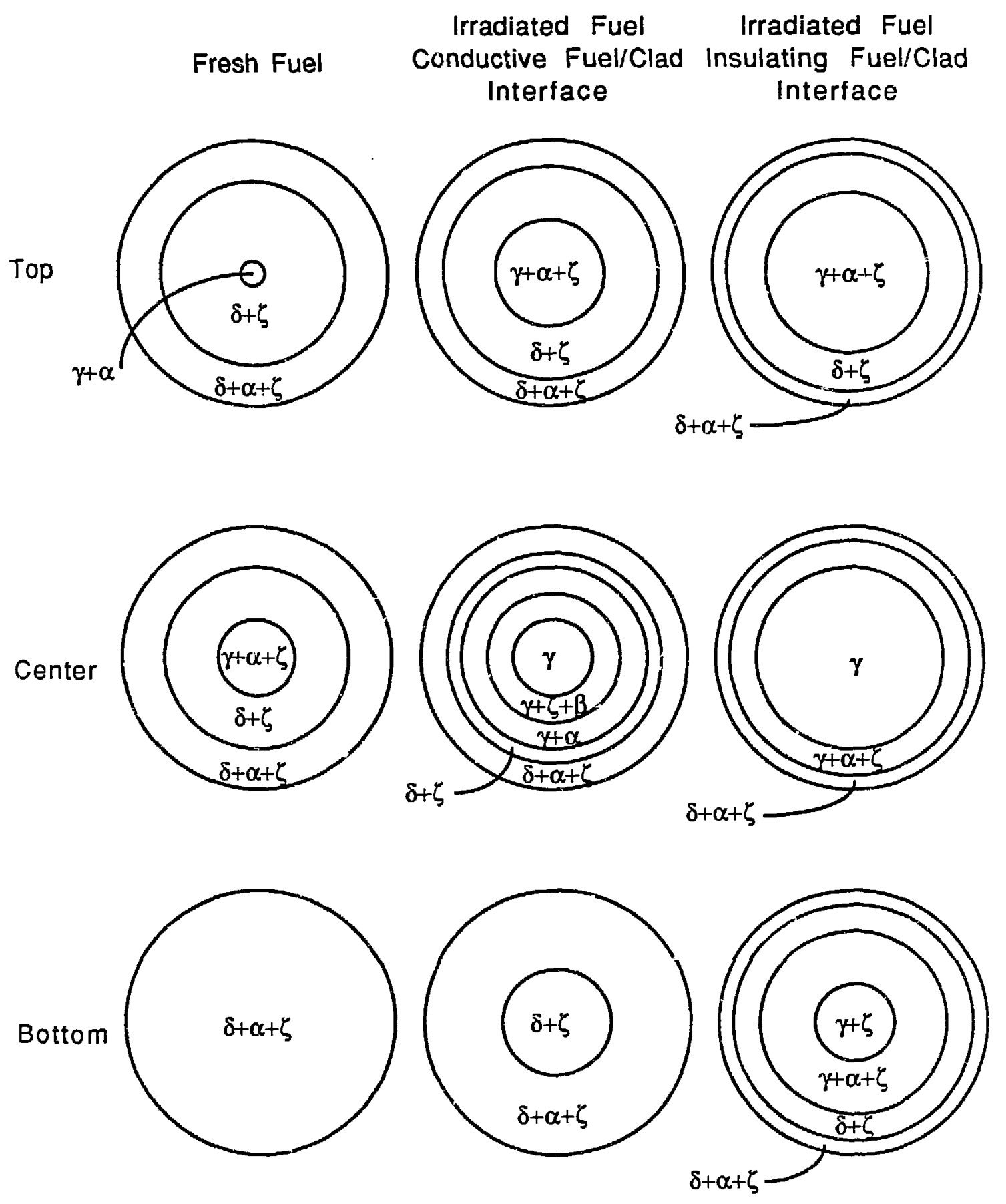

Figure 39. Possible phases and phase boundaries in U-19PU-10Zr, element T305. 

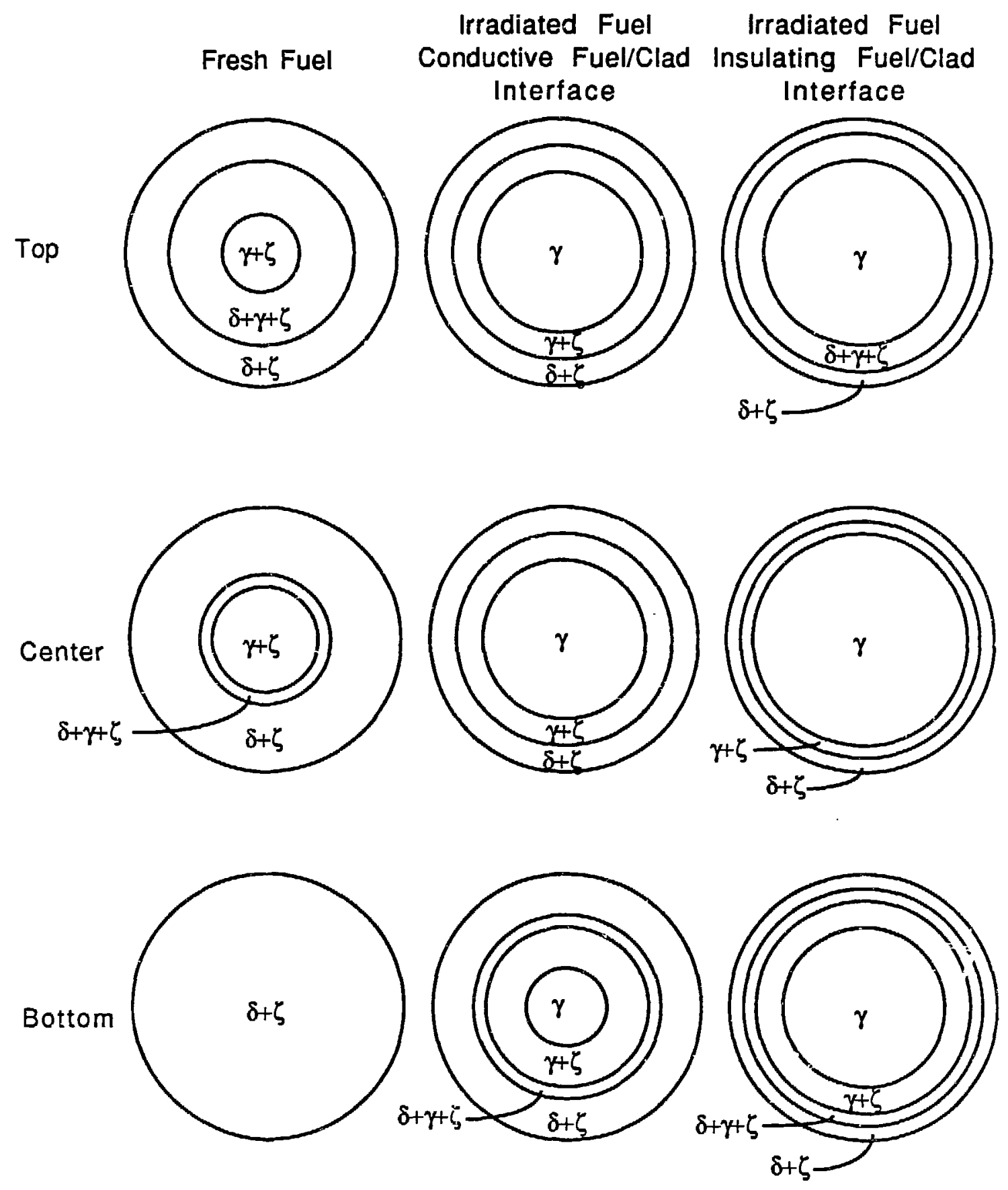

Figure 40. Possible phases and phase boundaries in U-22Pu-10Zr, element T303. 

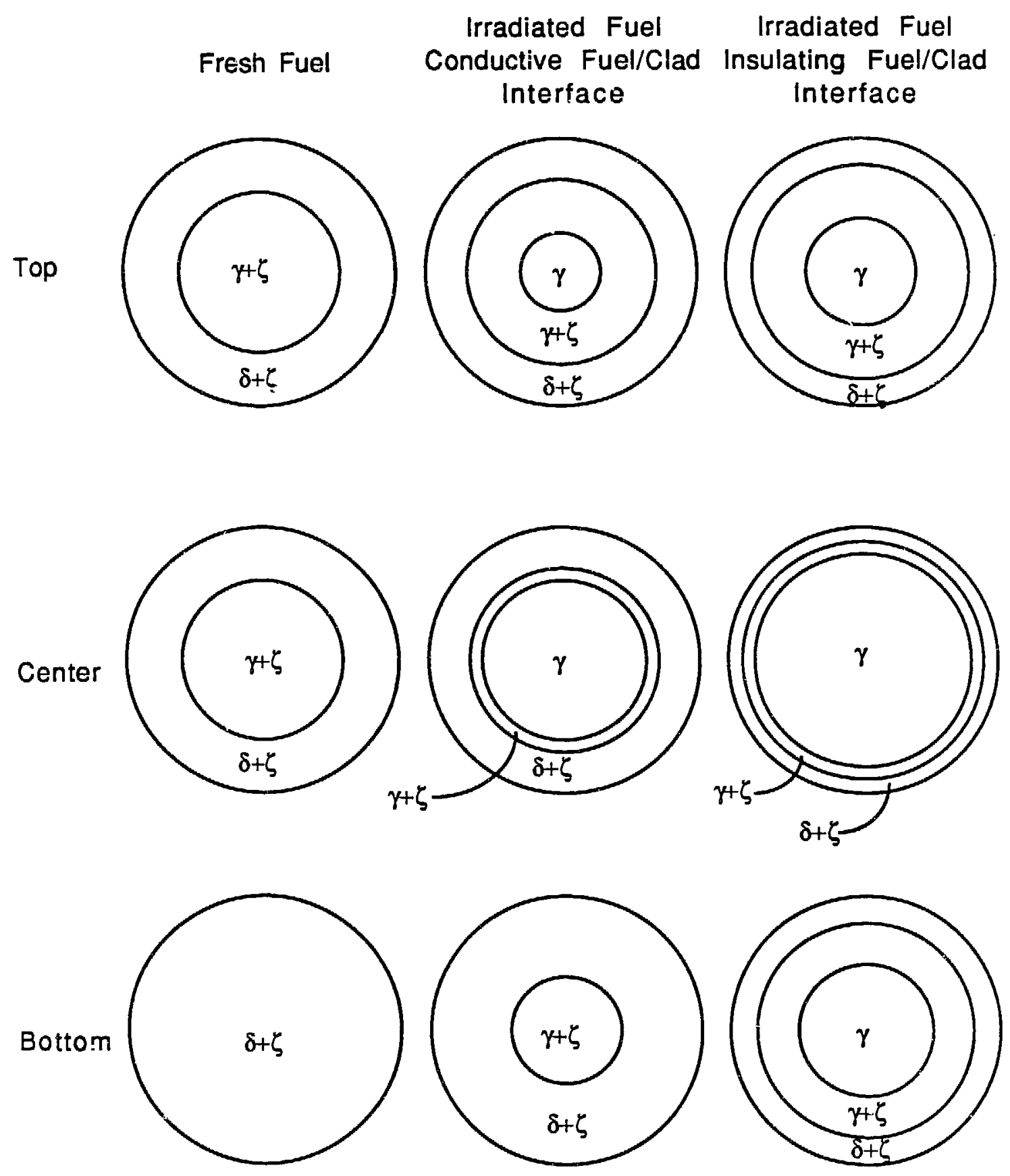

Figure 41. Possible phases and phase boundaries in U-26Pu-10Zr, element T353. 